ARTICLE

https://doi.org/10.1038/s41467-020-19328-1

\title{
Role of specialized composition of SWI/SNF complexes in prostate cancer lineage plasticity
}

Joanna Cyrta et al.

Advanced prostate cancer initially responds to hormonal treatment, but ultimately becomes resistant and requires more potent therapies. One mechanism of resistance observed in around $10-20 \%$ of these patients is lineage plasticity, which manifests in a partial or complete small cell or neuroendocrine prostate cancer (NEPC) phenotype. Here, we investigate the role of the mammalian SWI/SNF (mSWI/SNF) chromatin remodeling complex in NEPC. Using large patient datasets, patient-derived organoids and cancer cell lines, we identify $\mathrm{mSWI}$ SNF subunits that are deregulated in NEPC and demonstrate that SMARCA4 (BRG1) overexpression is associated with aggressive disease. We also show that SWI/SNF complexes interact with different lineage-specific factors in NEPC compared to prostate adenocarcinoma. These data point to a role for mSWI/SNF complexes in therapy-related lineage plasticity, which may also be relevant for other solid tumors.

\footnotetext{
${ }^{\#} \mathrm{~A}$ list of authors and their affiliations appears at the end of the paper.
} 
$\mathrm{P}$ rostate cancer $(\mathrm{PCa})$ is the second most commonly diagnosed cancer and the fifth cause of cancer-related death in men worldwide ${ }^{1,2}$. Although most men are effectively treated by local therapies (surgery and/or radiotherapy), some develop metastatic recurrence or present with metastases at initial diagnosis. The mainstay of treatment for metastatic $\mathrm{PCa}$ is androgen deprivation therapy (ADT), but resistance ultimately develops with progression to castration-resistant prostate cancer (CRPC), which typically harbors a "luminal" (adenocarcinoma) phenotype (CRPC-Adeno) with continued dependence on androgen receptor (AR) signaling ${ }^{3-5}$. Improved, more potent androgen receptor signaling inhibitors (ARSi) have been developed to treat patients that are not responsive to these therapeutics, yet acquired resistance to these drugs ultimately develops as well. In CRPC, indifference to AR signaling may manifest with a distinct histomorphology and expression of neural-like markers, leading to neuroendocrine or small cell prostate cancer (CRPC$\mathrm{NE}^{5-7}$. Approximately $10-20 \%$ of CRPC cases treated with ARSi display a neuroendocrine phenotype $e^{5,8,9}$. CRPC-NE no longer responds to ARSi and carries a dismal prognosis, with a mean overall survival of 12 months and no specific standard of care treatment options available ${ }^{10}$. There is mounting evidence that CRPC-Adeno can transdifferentiate to an AR-indifferent state through a mechanism of lineage plasticity under specific genomic conditions, including but not limited to TP53, RB1, and PTEN loss $4,11-13$. Epigenetic regulators, such as EZH2, are also critical in this process $4,12,13$. Although the mammalian Switch Sucrose NonFermenting (mSWI/SNF) complex is another major chromatin regulator well known for its role in physiological processes and frequently altered in cancer ${ }^{14-16}$, its putative implication in NEPC lineage plasticity is unknown.

Mammalian SWI/SNF complexes, also known as Brg/Brahmaassociated factor (BAF) complexes, are a heterogeneous family of ATP-dependent chromatin remodeling complexes composed of about 11-15 protein subunits and generally considered as positive mediators of chromatin accessibility ${ }^{16}$. These complexes are evolutionarily conserved in eukaryotes and required for normal embryonic development ${ }^{16,17}$. Specialized complex assemblies with distinct functions have been identified at different stages of embryogenesis and during tissue maturation ${ }^{18-22}$. Over $20 \%$ of human malignancies carry a genomic alteration involving at least one of the SWI/SNF subunit genes ${ }^{14-16}$, including malignant rhabdoid tumors ${ }^{23}$, synovial sarcoma ${ }^{24}$, small cell carcinoma of the ovary hypercalcemic type, ovarian clear cell carcinoma, endometrioid carcinoma, bladder cancer, renal cell carcinoma, and lung adenocarcinoma, among others ${ }^{14,23,25-27}$.

To date, SWI/SNF alterations have not been studied in the context of advanced PCa. In this study, we show that SWI/SNF composition is altered in the setting of CRPC-NE and that in contrast to many of the above-cited tumor types, SWI/SNF can have tumor-promoting functions in $\mathrm{PCa}$. We also provide evidence that SWI/SNF interacts with different lineage-specific partners throughout PCa transdifferentiation. Collectively, these findings suggest that specialized SWI/SNF complexes are associated with PCa disease progression and may play a role in therapy resistance.

\section{Results}

SWI/SNF subunit expression is altered in CRPC-NE. To define somatic mutation frequencies of genes encoding SWI/SNF subunits across the entire spectrum of $\mathrm{PCa}$, we conducted a comprehensive analysis of whole exome sequencing (WES) data from $600 \mathrm{PCa}$ patients representing a wide range of the disease spectrum, including 56 CRPC-NE cases (Fig. 1a, Supplementary Data 1, Supplementary Data 2, Supplementary Data 3). No recurrent SWI/SNF somatic mutations were observed and there was a low overall rate of point mutations and insertions/deletions in those genes (59 samples, $9.8 \%$ of all cases) (Fig. 1b). We observed an increased percentage of loss-of-heterozygosity (LOH) by hemizygous deletion or copy number neutral LOH (CNNL), in 27 out of 28 genes (significant for 15 genes, proportion test, alpha $=0.05)$, when comparing localized hormone treatmentnaïve PCa vs. CRPC-Adeno (Supplementary Fig. 1, Supplementary Data 1). A similar result was obtained when comparing localized hormone treatment-naïve PCa and CRPC-NE cases (26 out of 28 genes with higher $\mathrm{LOH}$ frequency in CRPC-NE). Conversely, there were fewer differences when comparing CRPCAdeno and CRPC-NE. A significant increase in the fraction of LOH in CRPC-NE as compared to CRPC-Adeno (proportion test, alpha $=0.05)$ was only noted for three genes: $B R D 7(51 \%$ vs. $30 \%$, respectively, $p=0.005)$, SMARCD1 ( $11 \%$ vs. $3 \%, p=0.04)$, and PBRM1 (18\% vs. $8 \%, p=0.049$ ) (Fig. $1 b$ ). However, this was not accompanied by a decrease in SMARCD1 or PBRM1 expression in CRPC-NE (Supplementary Fig. 2). Expression levels of BRD7 were significantly lower in CRPC-NE compared to CRPC-Adeno, but not in CRPC-NE compared to localized PCa. This is in line with a previous study in which BRD7 loss was identified as part of a larger heterozygous deletion event enriched in CRPC-NE and centered around the CYLD gene ${ }^{4}$. Collectively, these observations suggest that the increased fractions of $\mathrm{LOH}$ observed in CRPC-NE for BRD7, PBRM1, and SMARCD1 are unlikely to carry functional significance.

Given the modest differential abundance of genomic lesions, we next queried the expression levels of SWI/SNF subunits by examining RNA-seq data of 572 unique PCa patients, including 20 CRPC-NE cases $^{4,5}$ (Supplementary Data 4). The SMARCA4 ATPase subunit was significantly upregulated, with accompanying downregulation of its mutually exclusive paralogue SMARCA2 $2^{16,28}$ in CRPC-NE $(n=20)$ compared to CRPCAdeno $(n=120)$ with a mean difference of $0.55(p=0.015)$ (averaged $\log 2(\mathrm{FPKM}+1))$ for SMARCA4 and mean difference of $-0.60(p=0.02)$ for $S M A R C A 2$, respectively (Fig. 1c). A concordant result was observed when comparing SMARCA4/ SMARCA2 expression ratios per patient in CRPC-Adeno (median ratio $=1.07)$ and in CRPC-NE (median ratio $=3.06, p=0.007$ ) (Supplementary Fig. 3). To validate that these transcriptomic findings translated into differences in protein expression, we performed immunohistochemistry (IHC) on patient samples and confirmed higher SMARCA4 (BRG1) and lower SMARCA2 (BRM) expression with increasing PCa disease progression, with highest SMARCA4 expression observed in CRPC-NE (Fig. 1d and Supplementary Fig. 4).

Importantly, we also identified strong upregulation of neuronspecific SWI/SNF subunit genes in CRPC-NE: ACTL6B (BAF53B), DPF1 (BAF45B), and SS18L1 (CREST) (mean log2 $[$ FPKM +1$]$ values: $2.79,1.19$, and 3.58 , respectively) compared to CRPC-Adeno (mean $0.24, p=4.86 \mathrm{e}-06$; mean $0.35, p=$ 0.0016 ; and mean $2.76, p=6.85 \mathrm{e}-05$, respectively) (Fig. 1c). These subunits are expressed in post-mitotic neurons, serving instructive functions in neuronal differentiation ${ }^{22}$. By IHC, BAF53B, and BAF45B were highly expressed in CRPC-NE, but absent from benign prostate, localized PCa or CRPC-Adeno samples (Fig. 1d), demonstrating high specificity for the neuroendocrine phenotype.

We also noted intra-tumor heterogeneity in the expression of SWI/SNF subunits, as illustrated by IHC in patient specimens with a mixed phenotype (combining areas with adenocarcinoma and neuroendocrine differentiation) (Supplementary Figs. 4 and 5) and in 3D CRPC-NE organoid cultures (Supplementary Fig. 6). In the latter, we identified distinct cell clusters with high expression of the neural stem cell factor SOX2, low expression 
a
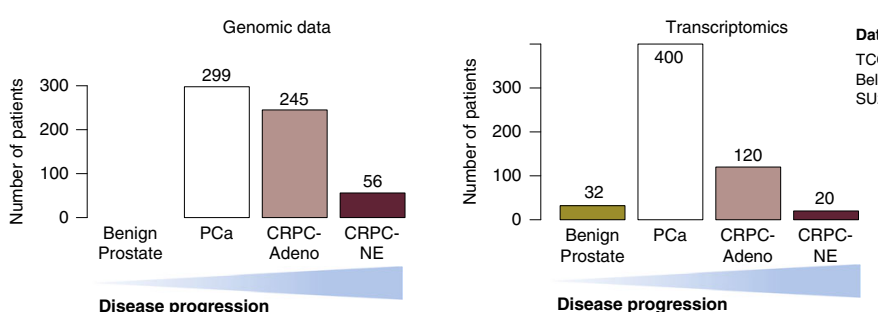

Disease progression

C

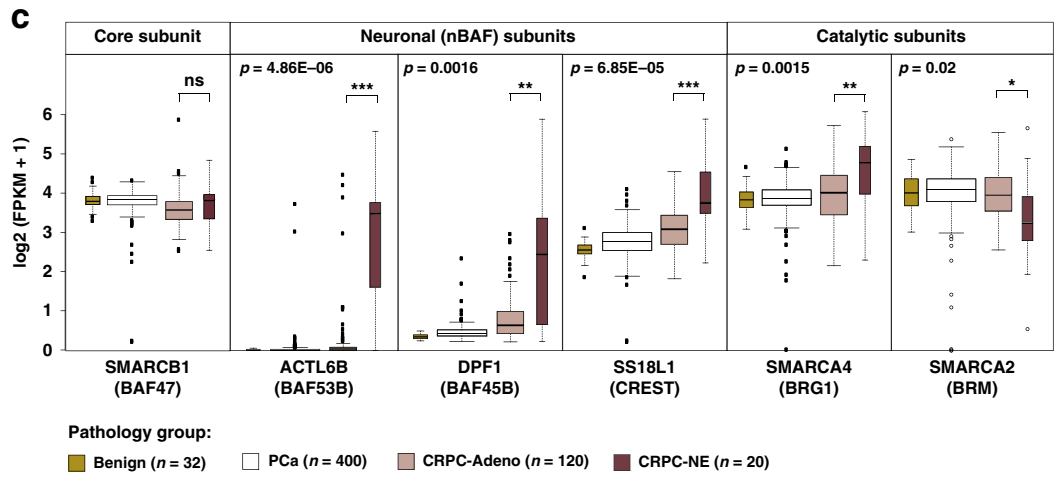

d
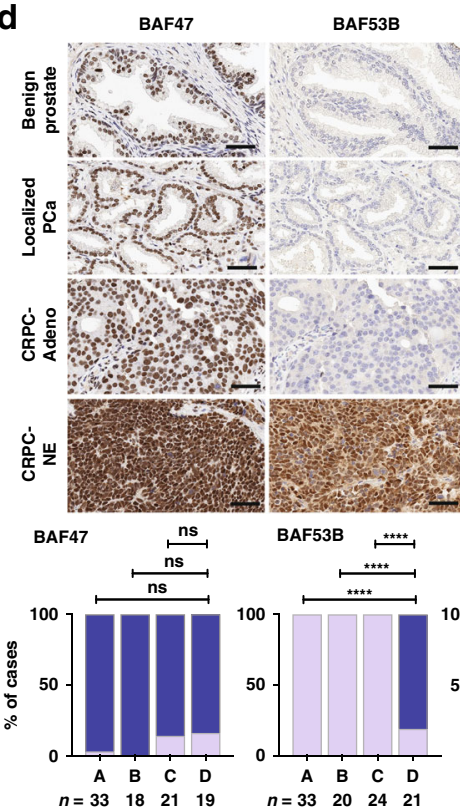
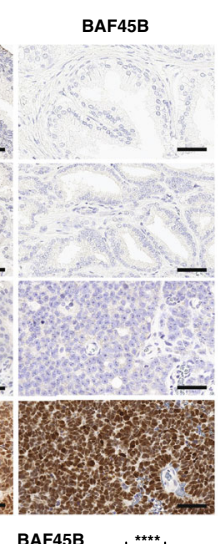

BRG1 (SMARCA4) BRM (SMARCA2)

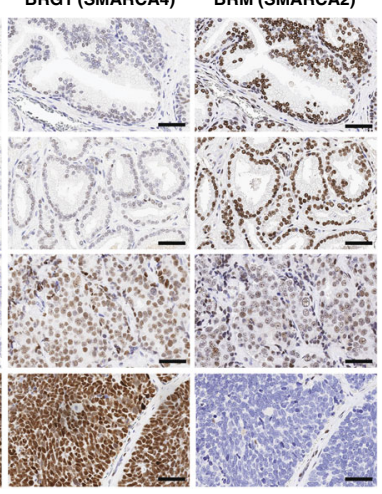

SMARCA4

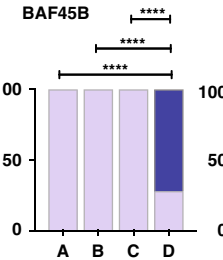

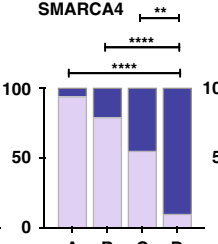

A $\quad$ B $C$ C $D$ $n=29-19-20 \quad 5$
Datasets Beltran, Nat Med 2016 SU2C-PCF, PNAS 2019 b

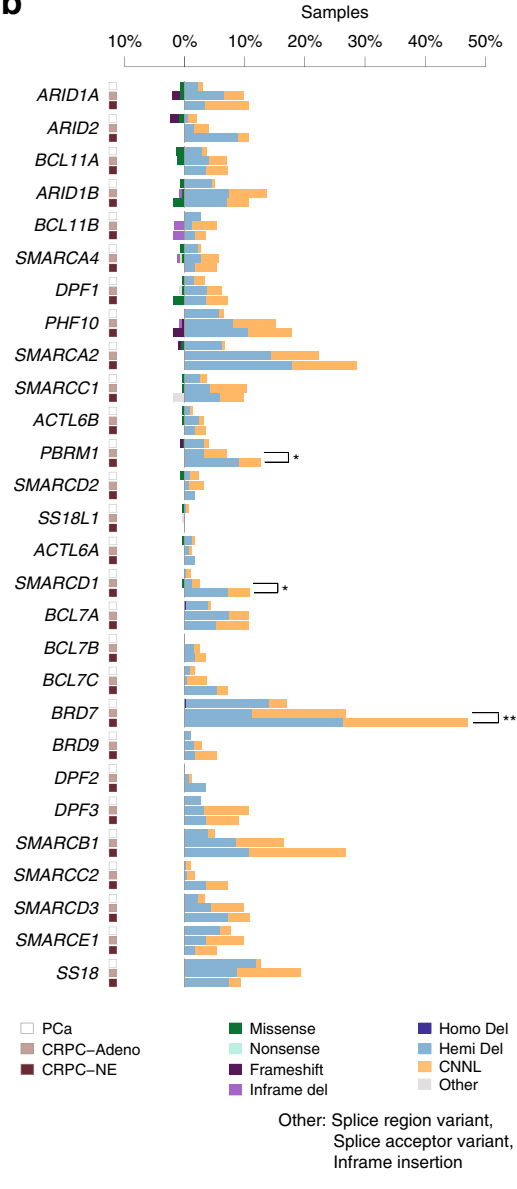

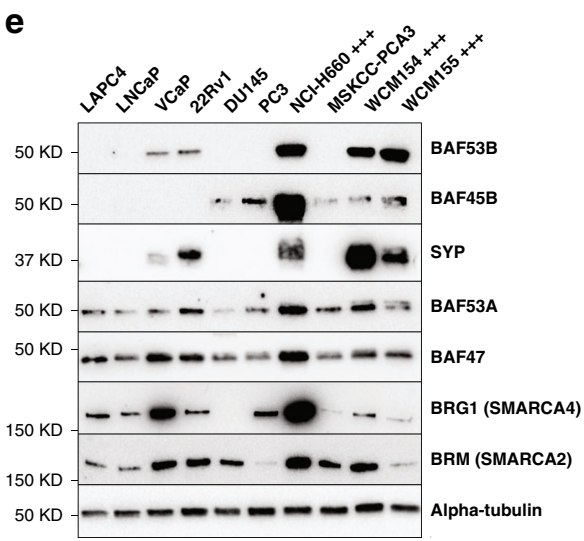

Fig. 1 Identification of SWI/SNF subunits deregulated in CRPC-NE. a Summary of the number of patients analyzed by whole exome sequencing (WES) and RNA-seq for each disease state. $\mathbf{b}$ WES results for SWI/SNF genes in 600 samples from unique PCa patients. For each gene, three consecutive bars represent alteration frequency in localized hormone treatment-naïve PCa, CRPC-Adeno and CRPC-NE, respectively. c RNA-seq analysis of gene expression levels in 572 unique patient samples from four studies, showing selected genes (ACTL6B: $p=4.86 \mathrm{E}-06$, DPF1: $p=0.0016$, SS18L1: $p=6.85 \mathrm{E}-05$, SMARCA4: $p=0.0015$, SMARCA2: $p=0.02$ ) significantly deregulated in CRPC-NE. The core subunit SMARCB1 is shown for comparison. The box plots represent the median values and the lower and upper interquartile range $(I Q R)$; the upper whisker $=\min (\max (x), Q 3+1.5 \times I Q R)$ and lower whisker $=\max$ $(\min (\mathrm{x}), \mathrm{Q} 1-1.5 \times I \mathrm{QR})$, and the outliers are plotted as individual points. d Representative immunostainings against BAF47 (SMARCB1), BAF53B (ACTL6B), BAF45B (DPF1), BRG1 (SMARCA4) and BRM (SMARCA2), and statistical analysis of staining intensity in patient samples. A-benign prostate glands, Bhormone treatment-naïve localized PCa, C-CRPC-Adeno, D-CRPC-NE. ${ }^{\star \star} p<0.01$ ( $p=0.0057$ for BRG1, $p=0.0012$ for BRM), ${ }^{\star \star \star} p<0.001(p=0.0004$ for $B R M)$ and ${ }^{\star \star \star \star} p<0.0001$, ns indicates not significant (two-sided Fisher's exact test). Scale bars, $50 \mu \mathrm{m}$. e Immunoblot showing expression levels of selected SWI/SNF subunits in PCa cell lines ( +++ designates CRPC-NE cell lines). Benign: benign prostatic tissue, PCa: localized hormone treatmentnaïve prostate cancer, CRPC-Adeno: Castration resistant prostate cancer, adenocarcinoma subtype, CRPC-NE: Castration resistant prostate cancer, neuroendocrine subtype. Source data are provided in the Source Data file. 
of the terminal neuronal marker synaptophysin, and higher expression of SMARCA4 (BRG1) and SMARCC1 (BAF155) than in the rest of the cell population (Supplementary Fig. 6). Overall, these observations suggest a relationship between expression of specific SWI/SNF subunits and different phenotype states, which can be seen even in a clonal tumor population.

BAF53B and BAF45B protein expression was confirmed in CRPC-NE cell lines and organoids (NCI-H660, WCM154, and WCM155 ${ }^{29}$ ) (Fig. 1e). BAF53B was also detected, albeit at lower levels, in two synaptophysin-positive PCa cell lines VCaP and 22Rv1, which bear some degree of transcriptomic similarity to neuroendocrine PCa cell lines ${ }^{9}$. BAF45B, on the other hand, was detected in some CRPC-Adeno cell lines and organoids (DU145, PC3, and MSKCC-PCA3). Unlike what we observed in patient samples, we did not observe high SMARCA4 (BRG1) and low SMARCA2 (BRM) expression in CRPC-NE cell lines, which could in part be due to marked differences in cell growth rates among different cell lines (Fig. 1e).

Although in neurons, BAF53B has been characterized as a mutually exclusive paralog to BAF53A, our data revealed that in CRPC-NE, BAF53A expression is maintained (Fig. 1e, Supplementary Fig. 7). BAF53B expression in neurons is known to be mediated by the downregulation of the RE1-Silencing Transcription factor (REST), a negative regulator of neuron-specific genes $^{20}$. In prostate adenocarcinoma cells, we observed that short-term REST knock-down led to an increase of BAF53B (ACTL6B) mRNA and protein levels, but the effect was modest, while other neuronal genes known to be negatively controlled by REST (e.g., synaptophysin) were highly upregulated (Supplementary Fig. 8).

To understand whether high SMARCA4 expression in CRPC$\mathrm{NE}$ was related to other characteristics of CRPC-NE, such as acquisition of pluripotent stem cell-like features, and not only to the expression of terminal neural markers, we performed singlecell RNA-seq on two CRPC-NE organoids in 3D culture (MSKCC PCa1 and 16) and confirmed that SMARCA4 expression was significantly higher in cells with high expression of the pluripotent stem cell marker SOX2 (Supplementary Fig. 9), consistent with our IHC findings (Supplementary Fig. 6). Bulk RNA-seq data from 18 PCa organoids (CRPC-Adeno and CRPCNE) (Supplementary Fig. 10) revealed that SMARCA4 expression was positively correlated with the expression of synaptophysin (a terminal neuronal marker), but also showed a tendency towards positive correlation with SOX2; conversely, there was a trend towards an inverse correlation between SMARCA2 and SOX2 (Pearson correlation analysis). Of note, some organoids (including MSKCC PCa1 and PCa16) classified as CRPC-NE based on their transcriptomic NEPC score ${ }^{4}$ showed high expression of SOX2, but low expression of terminal neural markers, such as synaptophysin (SYP) (Supplementary Fig. 10). These results suggest that high SMARCA4 expression may be related to pluripotent stem cell-like features and/or to proliferation at least in some CRPC-NE, rather than just to the expression of terminal neuronal markers.

Taken together, the above observations suggest that specialized SWI/SNF composition varies with PCa lineage plasticity to small cell or neuroendocrine states.

High SMARCA4 (BRG1) expression is associated with aggressive PCa. We posited that high SMARCA4 expression is associated with a more aggressive clinical course. To address this, we interrogated protein expression of SMARCA4 (BRG1) by IHC in a cohort of 203 men operated for localized hormone-treatment naïve PCa (demographics previously described in Spahn et al. ${ }^{30}$ ). High SMARCA4 protein expression in primary $\mathrm{PCa}$ was associated with a significantly shorter overall survival $(\mathrm{HR}=2.17$ [95\% CI: 1.07-4.42], $p=0.028$ ) (Fig. 2a). This relationship remained significant after adjustment for single covariates that have known association with PCa outcome (Supplementary Table 1). Patients with high tumor SMARCA2 (BRM) protein expression showed a trend towards a better overall survival, although this relationship did not reach statistical significance. Taken together, the above findings suggest that high SMARCA4 expression is associated with more aggressive cases of PCa.

We next sought to determine the effects of SMARCA4 and SMARCA2 depletion in PCa cell lines. We performed siRNAmediated knock-down of SMARCA4 and SMARCA2 in an androgen-sensitive (LNCaP) cell line and in a CRPC-Adeno cell line (22Rv1) and compared global transcriptional alterations using RNA-seq. As expected, given its posited dominant role, SMARCA4 depletion demonstrated a stronger effect on the transcriptome of both cell lines, while SMARCA2 depletion led to only modest transcriptional alterations (Fig. 2b, Supplementary Figs. 11 and 12). Among the genes most significantly deregulated upon SMARCA4 knock-down were several of known significance in PCa progression, including: upregulation of cell cycle regulators CDKN1A (p21) and BTG2 (in both LNCaP and $22 \mathrm{Rv} 1$ cell lines), downregulation of E2F targets (in both cell lines), downregulation of $E Z H 2$, and downregulation of the oncogenic long non-coding RNA PCAT-1 (both significant in LNCaP only) ${ }^{4,31,32}$ (Fig. 2c-e, Supplementary Fig. 12, Supplementary Data 5, Supplementary Data 6). We also observed a significant enrichment in gene sets related to EZH2 knock-down, suggesting that knock-down of SMARCA4 and knock-down of EZH2 can have partly overlapping effects in $\mathrm{PCa}$ cells (Supplementary Fig. 13). Expression of REST was not altered by SMARCA4 knock-down (Supplementary Fig. 14).

The observed changes in cell cycle-related pathways led us to explore the requirement for SMARCA4 and SMARCA2 for PCa cell growth. Depletion of SMARCA4, but not of SMARCA2, significantly reduced proliferation of the adenocarcinoma cell line LNCaP and the LNCaP-derived androgen-independent CRPCAdeno cell line C4-2 (Fig. 2f), in line with previous findings ${ }^{33,34}$. Knock-down of SMARCA4, but not of SMARCA2, in PCa cells resulted in a decrease of other SWI/SNF subunits, including SMARCC1 (BAF155) and ACTL6A (BAF53A), at the protein level, but not at the transcript level (Supplementary Fig. 15). Accordingly, both LNCaP and C4-2 cells proved to be highly sensitive to depletion of BAF155 (SMARCC1) (Supplementary Fig. 16). Recent work has shown that sensitivity of PCa cells to SMARCA4 knock-down may be dependent on PTEN loss, via a mechanism of synthetic lethality ${ }^{33}$. To expand upon these findings, we performed knock-down of BAF155 (SMARCC1) in two PTEN wild-type cell lines, 22Rv1 (CRPC-Adeno) and WCM154 (CRPC-NE), and observed a significant decrease in cell growth (Supplementary Fig. 17). This suggests that PTENcompetent PCa cells can still be sensitive to SWI/SNF disruption, even though they may be differentially responsive to depletion of different subunits.

Given that loss of TP53 and/or $R B 1$ has been suggested to confer a poised pluripotent state required for neuroendocrine transdifferentiation $^{11,12}$, we also tested the effect of SMARCA4 knock-down in LNCaP cells having undergone CRISPR-Cas9 mediated knock-out of TP53, RB1, or both genes. The effect of SMARCA4 knock-down on cell proliferation was not entirely abrogated by the absence of functional p53 and/or $\mathrm{Rb}$ (Supplementary Fig. 18).

To strengthen the above observations of a putative tumorpromoting function of SMARCA4 (BRG1) in PCa, we also sought to study the effects of SMARCA4 overexpression in PCa cells. 
a
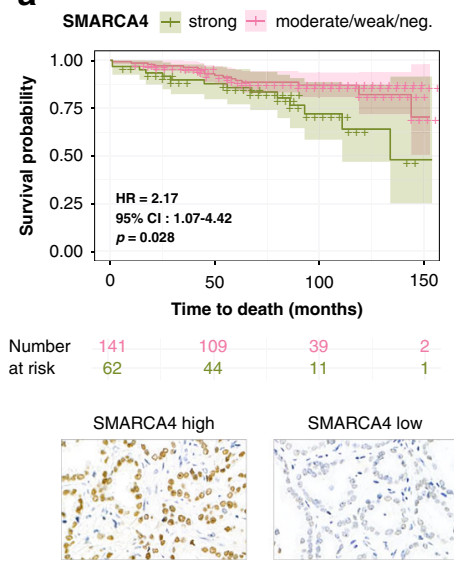

SMARCA2 + strong + moderate/weak/neg.

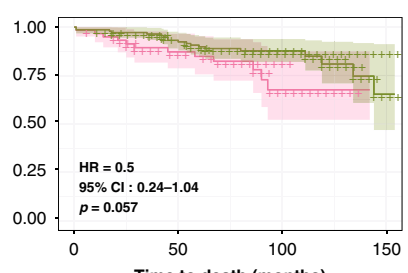

Time to death (months)

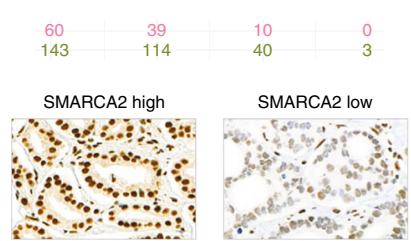

b

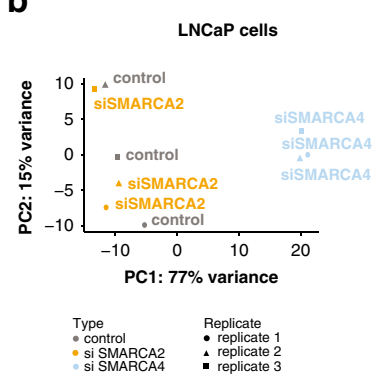

d
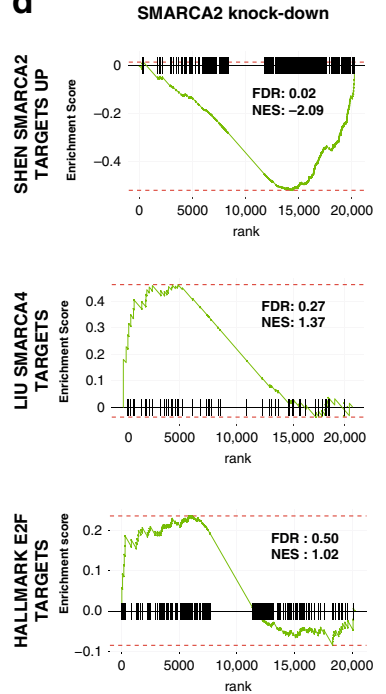

c

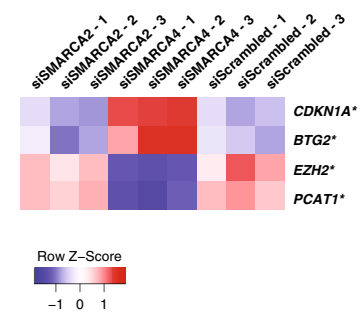

e
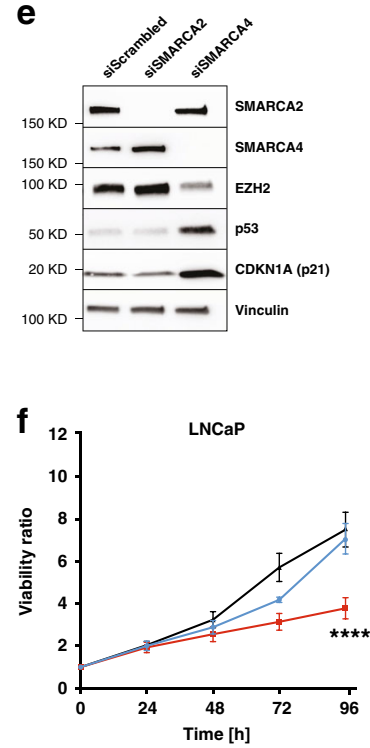

C4-2

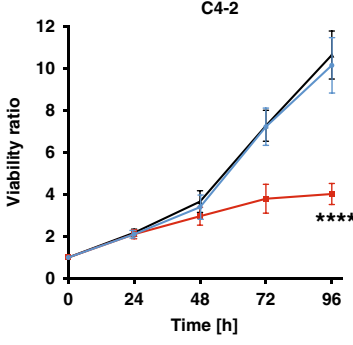

- si SMARCA4 $\rightarrow$ si SMARCA2

- si Scrambled

Fig. 2 SWI/SNF SMARCA4 and SMARCA2 expression in prostate cancer. a Kaplan-Meier curves showing the association between overall survival and SMARCA4 (BRG1) IHC expression ( $p=0.028$, Log-rank test) or SMARCA2 (BRM) IHC expression (not significant), in 203 patients with localized PCa. b Principal component analysis (PCA) of RNA-seq data for prostate adenocarcinoma (LNCaP) cells $72 \mathrm{~h}$ after SMARCA4 or SMARCA2 knock-down. c Expression levels (RNA-seq) of selected genes upon SMARCA4 and SMARCA2 knock-down in LNCaP cells; ${ }^{*}$ FDR $<0.05$. d Gene Set Enrichment Analysis based on RNA-seq gene expression analysis in LNCaP cells with SMARCA4 or SMARCA2 knock-down. e Immunoblot showing selected deregulated proteins upon SMARCA4 and SMARCA2 knock-down in LNCaP cells. f Effect of SMARCA4 or SMARCA2 knock-down on cell proliferation of prostatic adenocarcinoma (LNCaP) and CRPC-Adeno (C4-2) cells. $N=3$ independent experiments. Data are presented as mean values $+/-$ SEM and analyzed using two-way Anova $\left({ }^{\star \star \star *} p<0.0001\right)$. Statistical significance was evaluated at 0.05 alpha level with GraphPadPrism, version 8.2.1, Mac. Source data are provided in the Source Data file.

22Rv1 cells were stably transduced with lentiviral vectors designed to overexpress either SMARCA4 or SMARCA2 or with a matched empty control vector, and sorted based on the expression of the fluorescent reporter. Despite strong expression of the reporters, we did not observe an increase in SMARCA4 or SMARCA2 at the protein level (Supplementary Fig. 19). However, after an additional $24 \mathrm{~h}$ treatment with the proteasome inhibitor MG-132, SMARCA4, and SMARCA2 overexpression was readily detected. These findings hint towards a tight and contextdependent regulation of catalytic SWI/SNF subunits, as forced isolated overexpression of a single subunit seems to provoke rapid degradation of the excess protein. Thus, it is possible that SMARCA4 overexpression may be necessary, but not sufficient, to promote an aggressive phenotype in prostate cancer cells.

To understand whether BAF53B and BAF45B-two other subunits overexpressed in CRPC-NE-potentially regulated similar gene expression programs as SMARCA4, we performed shRNA-mediated knock-down of these subunits in the CRPC-NE organoid line WCM155. Neither BAF53B nor BAF45B knockdown had an effect on CRPC-NE cell proliferation (Supplementary Fig. 20) Therefore, it appears that BAF53B and BAF45B expression may be specific for the CRPC-NE phenotype, but not a critical mediator of CRPC-NE aggressiveness. Collectively, the above genomic, transcriptomic, and functional findings support a tumor-promoting role of SMARCA4-containing mSWI/SNF complexes in PCa.

Aggressive prostate cancer anti-correlates with SMARCA4 knock-down signature. Based on the association of SMARCA4 expression with poor clinical outcome, and observations that SMARCA4 knock-down leads to a significant decrease in PCa cell growth in line with previous studies ${ }^{33,34}$, we posited that a SMARCA4 knock-down signature (composed of genes deregulated upon SMARCA4 depletion) would be associated with more indolent PCa. To address this, we interrogated RNA-seq data of several large clinical cohorts using a SMARCA4 knock-down signature derived from the LNCaP PCa cell line (see "Methods" section) and composed of the top 419 deregulated genes. A high SMARCA4 knock-down signature score was, indeed, associated with more indolent disease. In contrast, a low SMARCA4 knockdown signature score was associated with more aggressive PCa.

As expected, a low SMARCA4 knock-down signature score was also strongly associated with a CRPC-NE phenotype. We 
a
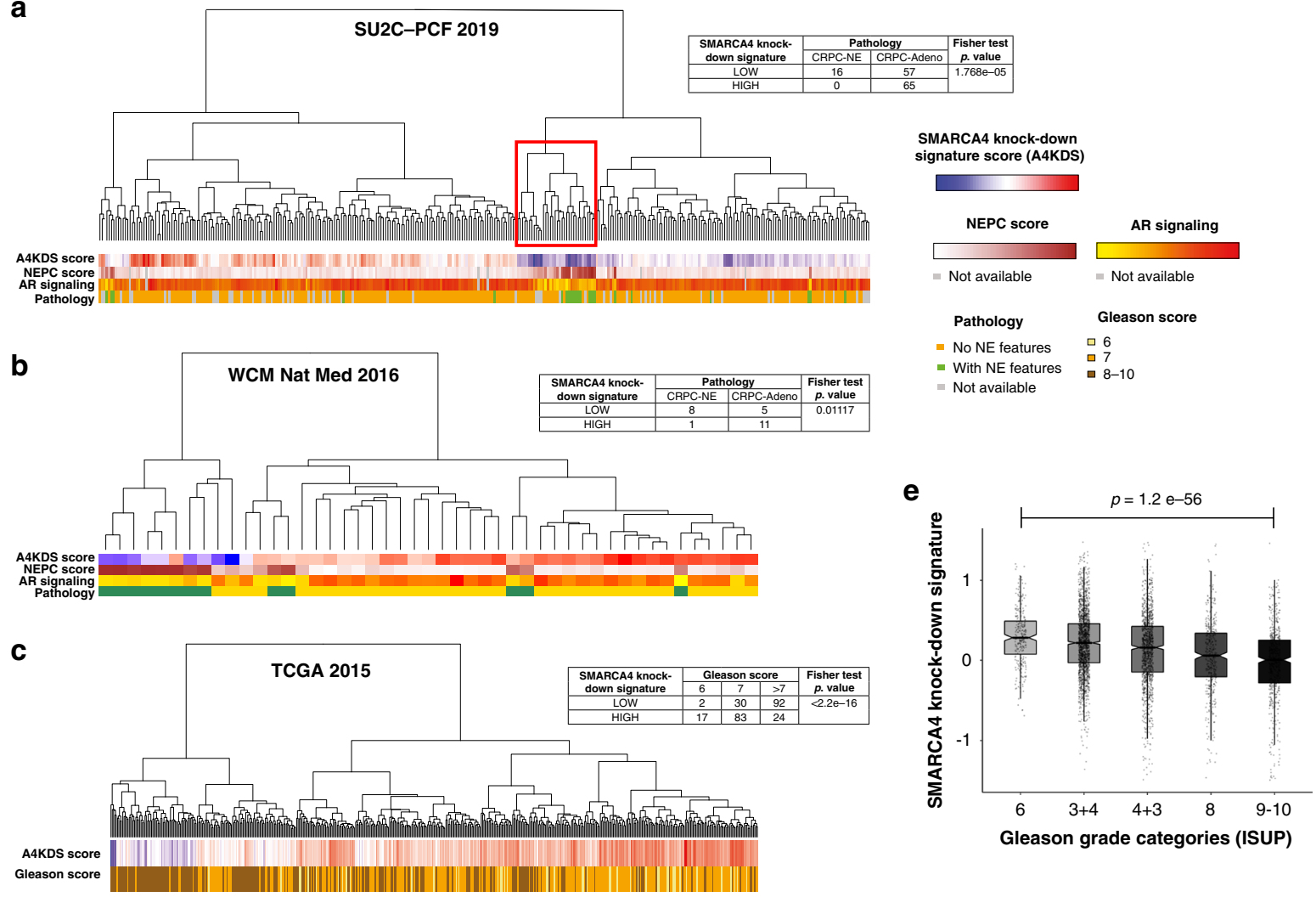

d
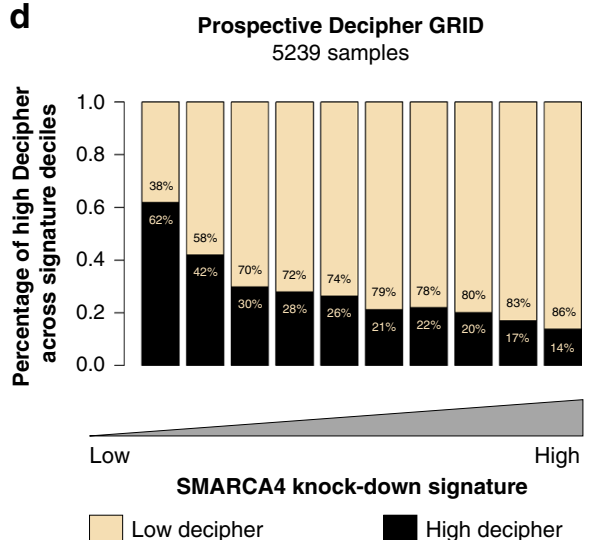

f

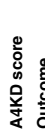

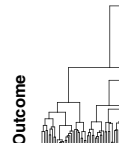
JHMI JHMI
355 samples
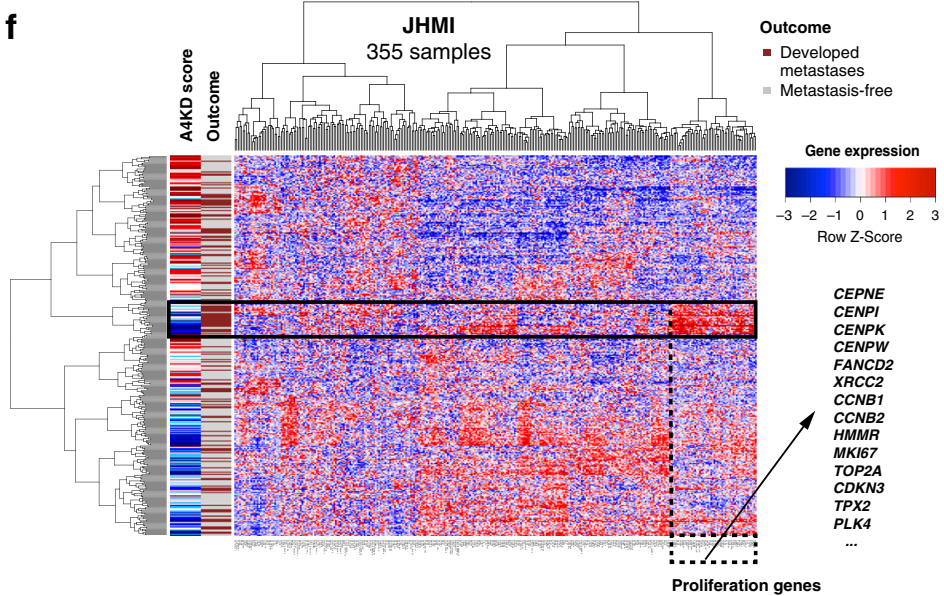

Fig. 3 Transcriptomic SMARCA4 knock-down signature in PCa cohorts. a 332 cases of CRPC from the SU2C-PCF cohort (table: $n=138$ ). b 47 cases of CRPC from the WCM cohort (table: $n=25$ ). c 495 cases of localized PCa from the TCGA cohort (table: $n=248$ ); $p$ value $=1.21$ e-18 (a-c represent twopaired tests). d Low SMARCA4 knock-down signature scores are associated with high Decipher scores (surrogate for risk of metastasis) in 5239 primary PCa samples from the Prospective Decipher GRID (Mann-Whitney U test). e Low SMARCA4 knock-down signature scores are associated with higher Gleason score in the same Decipher GRID cohort (Mann-Kendall trend test). The center of each boxplot represents the mean, lower bound represents the 25th percentile, the top bound represents the 75th percentile, the whiskers represent the $95 \% \mathrm{Cl}$. $p$ value $=1.2 \mathrm{e}-56$. f Unsupervised clustering of patients from in the JHMI natural history PCa cohort (Johns Hopkins Medical Institute, $n=355$ ) based on the downregulated genes from the SMARCA4 knockdown signature, and compared to metastatic outcome (brown: metastatic recurrence, gray: metastasis-free). Overexpression of a subset of genes, many of which are related to proliferation, is seen in a cluster of patients who presented metastatic outcome (black box).

examined two CRPC cohorts consisting of 332 patients from the Stand Up To Cancer-Prostate Cancer Foundation (SU2C-PCF) trial treated with $\mathrm{ARSi}^{5}$ and 47 patients from the Weill Cornell Medicine (WCM) cohort ${ }^{4}$. In the SU2C-PCF cohort, when considering patients from the highest (top 25\%) and lowest (bottom 25\%) quartiles of SMARCA4 knock-down signature scores $(n=138)$, low SMARCA4 knock-down signature scores were significantly more often observed in CRPC-NE cases $(n=16$ or $100 \%)$ than in CRPC-Adeno cases $(n=57$ or $46.7 \%)(p=$ 1.77e-05) (Fig. 3a). A similar result was obtained in the WCM cohort $(n=25)$ : low SMARCA4 knock-down signature scores were seen in $89 \%(n=8)$ of CRPC-NE cases vs. $31 \%(n=5)$ of CRPC-Adeno cases $(p=0.011)$ (Fig. 3b). Furthermore, low SMARCA4 knock-down signature was associated with a higher NEPC $^{4}$ and a lower AR signaling score ${ }^{35}$ in both cohorts (Supplementary Table 2). One particularly informative cluster 
was found to show low SMARCA4 knock-down signature scores, high CRPC-NE scores, and low AR signaling scores (Fig. 3a, red box). Of note, SMARCA4 mRNA levels were consistent with the predicted signature score in all analyzed cohorts (Supplementary Fig. 21).

We next queried if the SMARCA4 knock-down signature was associated with higher tumor grade, referred to as Gleason score risk groups in localized $\mathrm{PCa}^{36}$. We first explored 248 patients from The Cancer Genome Atlas (TCGA) PCa cohort with localized, hormone treatment-naive $\mathrm{PCa}^{37}$. Tumors in the highest Gleason score risk groups (IV and V) more often displayed low SMARCA4 knock-down signature scores $(p<2.2 \mathrm{e}-16)$ (Fig. $3 \mathrm{c}$ ).

As high tumor grade is associated with risk of metastatic progression, we decided to validate these findings in other independent clinical cohorts annotated with clinical survival data. We calculated SMARCA4 knock-down signature scores for 5239 prospectively collected radical prostatectomy samples from men with localized PCa and analyzed with the Decipher GRID transcriptomic platform ${ }^{38}$. Samples with a low SMARCA4 knockdown signature (lowest 10\%) were significantly enriched (62\%) with high Decipher score, which is a strong surrogate of metastasis prediction ${ }^{38}$ (Fig. 3d), compared to $14 \%$ in samples with high SMARCA4 knock-down signature (highest 10\%). In this patient population and consistent with TCGA results, we observed an association between SMARCA4 knock-down signature and Gleason score risk categories: signature scores in the Gleason 9-10 group (mean $=-0.13$ ) were significantly lower compared to the Gleason 6 group (mean $=0.29, p=1.2 \mathrm{e}-56$ ) (Fig. 3e). We next explored an independent retrospective cohort from Johns Hopkins Medical Institution (JHMI) ${ }^{39}$. In the JHMI cohort, patients with low SMARCA4 knock-down signature showed a trend towards higher metastasis frequency, the strongest surrogate for lethal disease progression (Supplementary Fig. 22). When clustering patients based on the downregulated genes (Fig. 3f) or on all genes (Supplementary Fig. 23) that make up the SMARCA4 knock-down signature, overexpression of a subset of genes involved in cell proliferation was associated with a cluster of patients enriched with metastatic outcome (Fig. 3f, box). In summary, these results from large patient cohorts confirm that the lowest SMARCA4 knock-down signatures are observed in the most aggressive PCa.

The SWI/SNF complex has distinct lineage-specific interaction partners in CRPC-NE and in prostate adenocarcinoma cells. To gain insight into the potential effectors of NEPC-specific epigenetic regulation, we next sought to identify interactors of $\mathrm{mSWI} /$ SNF in the context of CRPC-NE and prostate adenocarcinoma cell lines. To this end, we performed co-IP with an antibody directed against the core SWI/SNF subunit BAF155 (SMARCC1) at low stringency (see "Methods" section) followed by mass spectrometry (MS) in NCI-H660 (a CRPC-NE cell line) and in LNCaP-AR cells (LNCaP cells engineered to overexpress the androgen receptor ${ }^{40}$ ). Proteins that immunoprecipitated with BAF155 in CRPC-NE cells, but not in adenocarcinoma cells, (Fig. 4a, b) included BAF53B (ACTL6B) and BAF45B (DPF1) subunits, as anticipated from results described above, as well as several factors specific to neural differentiation, such as the transcription factor NKX2.1 (TTF-1), the microtubule-associated factor MAP2 and the growth factor VGF. Moreover, we found several members of the NuRD chromatin remodeling complex, such as MTA1 and CHD4, to immunoprecipitate with BAF155. This is in line with previous findings of a potential interaction of those two chromatin remodeling complexes (Fig. 4a, b) ${ }^{41,42}$. A considerable amount of CRPC-NE specific SWI/SNF interactors were proteins involved in chromatin regulation or DNA repair
(Fig. 4a, b, Supplementary Data 7, Supplementary Data 8). Conversely, proteins that immunoprecipitated with BAF155 in adenocarcinoma cells, but not in CRPC-NE, included HOXB13, a homeobox transcription factor involved in $\mathrm{AR}$ signaling ${ }^{43}$ (Fig. 4b). In line with these findings, genes encoding most of the above factors were differentially expressed between CRPC-NE and adenocarcinoma cell lines and organoids (Fig. 4c, Supplementary Fig. 24). Further, we confirmed unique interaction of factors NKX2.1, CHD4, MTA1, and VGF with BAF155 in NCI$\mathrm{H} 660$ by immunoblotting, while these interactions were absent in LNCaP-AR cells (Supplementary Fig. 25a). Interaction of HOXB13 with BAF155 in LNCaP-AR cells was also confirmed by immunoblotting (Supplementary Fig. 25b). The co-IP experiment also showed an enrichment of proteins negatively associated with REST signaling in NCI-H660 cells, such as HMG20A, a chromatin-associated protein known to overcome the repressive effects of REST and induce activation of neuronal genes ${ }^{44}$. Loss of expression or altered splicing of REST has been associated with neural-like lineage plasticity in PCa in multiple studies ${ }^{45-51}$. An independent co-IP experiment using an antibody directed against SMARCA4 followed by MS in NCI-H660 and in LNCaP cells found similar results for BAF53B, BAF45B, NKX2.1, and HOXB13 (Supplementary Fig. 26, Supplementary Data 9).

As a proof-of-principle, we compared genome occupancy of SMARCC1, HOXB13, the active chromatin histone mark $\mathrm{H} 3 \mathrm{~K} 27 \mathrm{ac}$ and the inactive chromatin mark H3K27me3 in LNCaP cells, using published ChIP-seq datasets (Supplementary Fig. 27). SMARCC1 and HOXB13 colocalized at active chromatin sites (11,824 sites), while there was almost no overlap between SMARCC1 and HOXB13 at inactive chromatin sites, thus suggesting a functional nature of this interaction. Collectively, the above observations suggest that the set of SWI/SNF interaction partners in CRPC-NE is quite distinct from the one in prostatic adenocarcinoma.

\section{Discussion}

Whereas neuroendocrine PCa is rarely present at diagnosis in hormone-treatment naïve PCa patients (de novo neuroendocrine $\mathrm{PCa},<1 \%$ of cases $)^{52}$, recent work supports the hypothesis that acquisition of a CRPC-NE phenotype in $\mathrm{PCa}$ is a more common mechanism of resistance to $\mathrm{ARSi}^{4,5,8,13,53}$. Based on a recent review of 440 CRPC patients, CRPC-NE was seen in $11 \%$ of CRPC patients that underwent biopsy ${ }^{5,8,9}$. There is increasing evidence that CRPC-NE can directly arise from CRPC-Adeno cells through lineage plasticity (Supplementary Fig. 28), which is supported by lineage tracing experiments in a genetically engineered mouse model of PCa with combined Trp53 and Pten loss $^{54}$. Moreover, mouse models with $\operatorname{Trp53}$ and $R b 1$ genomic loss show lineage plasticity, but epigenetic therapy can resensitize those tumors towards $\mathrm{ARSi}$ treatment ${ }^{11}$. In patient cohorts, CRPC-NE are characterized by an overexpression of several epigenetic regulators (such as EZH2) and a specific DNA methylation profile ${ }^{4,13,29}$. Overall, these data support the idea that PCa progression through lineage plasticity is regulated by epigenetic changes in a specific genomic context ${ }^{12,55}$.

Given that $\mathrm{mSWI} / \mathrm{SNF}$ complexes are major epigenetic regulators in physiological cell differentiation, we posited that they may play a role in CRPC-NE lineage plasticity. Specialized assemblies of the SWI/SNF complex with distinct functions are observed at different stages of embryonic development and tissue maturation ${ }^{18,19}$. The most notable changes in SWI/SNF composition described to date occur during neuronal differentiation. Cells committed to the neural lineage initially express a neural progenitor form of the complex (termed npBAF), which incorporates among others the BAF53A, BAF45A/D, and 

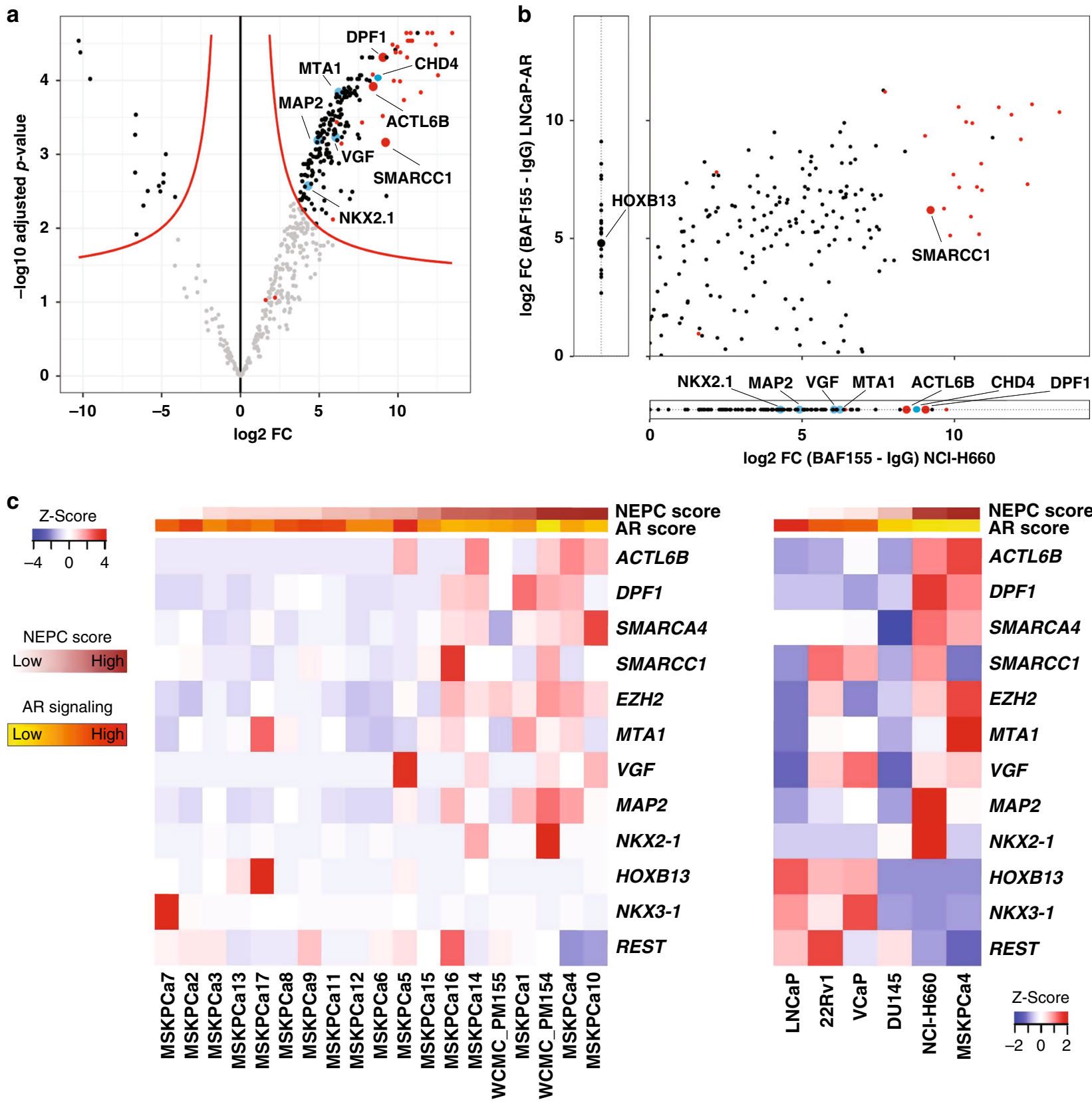

Fig. 4 SWI/SNF associates with different transcriptional regulators in CRPC-NE and in adenocarcinoma cells. a Volcano plot showing proteins most significantly represented (upper right) in the co-IP using an anti-BAF155 antibody, as compared to IgG isotype control in NCI-H660 (CRPC-NE) cells (pooled data from 3 co-IP replicates). The $x$-axis represents log2 fold change (FC) values, the $y$-axis represents $-\log 10$ of adjusted $p$-values. Each dot represents a protein; red dots represent SWI/SNF members, blue dots indicate notable findings. b A qualitative representation comparing proteins associated with SWI/SNF in NCl-H660 (CRPC-NE) and in LNCaP-AR (adenocarcinoma) cells (averaged data from two co-IP experiments). Plotted are $\log 2$ fold change values between BAF155 IP and IgG IP in NCI-H660 cells ( $x$-axis) and in LNCaP-AR cells ( $y$-axis), for proteins present in both cell lines with sufficient evidence in each cell line (i.e., if present in two replicates of at least one condition). Proteins plotted outside of the main field represent proteins that were detected exclusively in one of the cell lines. $\mathbf{c}$ Heatmap showing RNA-seq expression (FPKM) of prostate cancer 3D organoids (left) and 2D cell lines (right), ordered by increasing NEPC score.

SS18 subunits ${ }^{20-22}$. However, upon differentiation to post-mitotic neurons, the complex undergoes a switch to the neural variant and incorporates the respective paralogs of these subunits (i.e., BAF53B, BAF45B/C, and SS18L1). This switch is mediated by repression of BAF53A by micro-RNAs in response to downregulation of $\mathrm{REST}^{20}$. In this study, we observed for the first time the presence of "neuronal" SWI/SNF subunits outside of the nervous system, characterized by the expression of BAF53B and BAF45B in CRPC-NE. Although expression of these subunits was highly specific of CRPC-NE, it remains unclear whether they play a role in activating neural-like gene programs, or are simply expressed as a consequence of this process. Additional studies are warranted to assess the putative utility of BAF53B and BAF45B as CRPC-NE biomarkers or as predictors of patients at risk of developing CRPC-NE from CRPC-Adeno while on ARSi. Of note, expression of the BAF53A paralogue is retained in CRPC$\mathrm{NE}$, pointing to potential differences in the way SWI/SNF complexes assemble in post-mitotic neurons and in neuroendocrine 
cancer cells, and to possible co-existence of different forms of the complex within the same tumor.

This study supports a pleiotropic role for the SWI/SNF chromatin remodeling complex in cancer, which may depend on the genomic and/or the epigenetic context-a paradigm which has been gaining support both in regards to SWI/SNF and to other epigenetic regulators ${ }^{56-58}$. Although the complex has been described as a tumor suppressor in many cancer types ${ }^{14,23,25,59}$, there is increasing evidence for possible tumor-promoting functions of SWI/SNF in other malignancies, including leukemia, breast, liver and pancreas cancer melanoma, glioblastoma, neuroblastoma and synovial sarcoma ${ }^{24,60-65}$. In PCa, the role of SWI/ SNF has long remained insufficiently characterized. Our study provides novel evidence that it can have tumor-promoting functions in PCa, including its most aggressive forms. Based on prior studies and on the current analysis, mutations in SWI/SNF genes are very rare in $\mathrm{PCa}^{4,5,34,37,66-68}$ (see Fig. 1b), in contrast to some other cancers types ${ }^{14,15}$. From the functional perspective, inhibition of the SWI/SNF subunits BAF57 (SMARCE1) or BAF53A (ACTL6A) in PCa cells has been shown to abrogate androgen-dependent cell proliferation ${ }^{69,70}$. Similarly, Sandoval et al. reported that SWI/SNF interacts with ERG in PCa cells harboring the TMPRSS2:ERG gene fusion and is required to activate specific gene programs to maintain cell growth ${ }^{71}$. Although on the contrary, Prensner et al. had suggested that SWI/ $\mathrm{SNF}$ acts as a tumor suppressor in $\mathrm{PCa}$, by demonstrating an antagonistic relationship between the pro-oncogenic long noncoding RNA SChLAP1 and the SWI/SNF core subunit BAF47 72 , a subsequent study failed to confirm that SChLAP1-SWI/SNF interaction leads to depletion of SWI/SNF from the genome ${ }^{73}$. Most recently, two studies demonstrated that SMARCA4 was required for growth of prostatic adenocarcinoma cells ${ }^{33,34}$, as also confirmed by our results (Fig. 2). Accordingly, localized PCa has been reported to show higher SMARCA4 and lower SMARCA2 expression than benign prostate tissue ${ }^{33,34,74,75}$. We confirm these results and further report an overexpression of SMARCA4 in CRPC and especially in CRPC-NE, in contrast to lower expression in early PCa. In addition, we show that a low SMARCA4 knock-down gene signature score is associated with aggressive PCa, and with a CRPC-NE phenotype.

Recent work by Ding et al. specifically proposed a synthetic lethal association between PTEN and SMARCA4 in PCa, identified through a CRISPR-Cas9 screen $^{33}$. They showed that in vitro, SMARCA4 knock-down leads to decreased cell proliferation in PTEN-negative cell lines, and confirmed these findings in a mouse model. In our study, knock-down of the core SWI/SNF subunit BAF155 (SMARCC1) and BAF170 (SMARCC2) inhibited growth of both PTEN-deficient and PTEN-competent PCa adenocarcinoma cells (Supplementary Figs. 16 and 17), and the PTEN-competent CRPC-NE cell line WCM154 was sensitive to ablation of BAF155, but not of BAF170. This suggests that even if PTEN-competent cells are not sensitive to SMARCA4 loss, they may still be vulnerable to SWI/SNF disruption through depletion of other critical subunits. Taken together, our and previously published findings indicate that PCa expands the spectrum of cancer types in which SWI/SNF can display tumor-promoting functions.

In addition, we observed that SWI/SNF composition in prostate cancer is not a hard-set feature; instead, specialized forms of SWI/SNF may assemble in cancer cells depending on their phenotype (Fig. 5). There is increasing evidence that de-repression of "terminal" neuronal genes in PCa cells is not sufficient to model other critical steps of neuroendocrine lineage plasticity in CRPC$\mathrm{NE}^{76}$. As such, the distinct phenotype of CRPC-NE is not limited to the expression of terminal neuronal markers, but involves other key characteristics, such as dedifferentiation, AR signaling indifference, acquisition of stem cell-like features and/or high proliferation $^{13}$. In line with this, we show that some patientderived PCa organoids that are classified as CRPC-NE using a transcriptome-based NEPC score ${ }^{4}$ (Supplementary Fig. 10), do not all show high expression of terminal neural markers such as synaptophysin, but instead may highly express factors related to "stemness" (e.g., SOX2). Based on our observations, it is possible that specific forms of SWI/SNF are implicated in various abovementioned cellular processes, rather than only in the expression of terminal neuronal markers. One possible hypothesis is that an equivalent of the embryonic stem cell form of the complex (esBAF), which is known to exclusively incorporate BRG1 (SMARCA4), BAF53A and BAF155 (SMARCC1) subunits and not their paralogs ${ }^{18,19}$, could exist in cancers cells with pluripotent stem cell-like features, and possibly explain the overexpression and/or the functional requirement for these subunits. Similarly, neural-like forms of the complex, including BAF53B and/or BAF45B, could be more specific of cancer cells with a more terminal neural-like phenotype. Further studies are needed to determine whether variants of SWI/SNF can co-exist within the same cell or whether they define distinct tumor sub-populations, in line with what we have observed in 3D CRPC-NE organoid cultures (Supplementary Fig. 6).

One of the ways in which SWI/SNF might contribute to CRPCNE transdifferentiation is by cooperating with other transcriptional regulators in a context-dependent manner. To this end, we showed that SWI/SNF interacts with different lineage-specific proteins in CRPC-NE than in adenocarcinoma cells (Fig. 4b, Supplementary Fig. 25). In particular, SWI/SNF interacts with the transcription factor NK2 homeobox 1 (NKX2.1/TTF-1) in CRPCNE cells, but not in adenocarcinoma cells (Fig. 4c, Supplementary Fig. 25). TTF-1 is a master regulator critical for the development of lung and thyroid, but also of specific parts of the brain ${ }^{77-79}$ and is known to be expressed in neuroendocrine neoplasms, including CRPC-NE ${ }^{76}$. We also observed SWI/SNF interaction with Metastasis-associated Protein 1 (MTA1), a member of the nucleosome-remodeling and deacetylation complex (NuRD), which is overexpressed in metastatic prostate cancer ${ }^{80}$ (Fig. 4c). Conversely, we found HOXB13 to be specifically associated with SWI/SNF in adenocarcinoma cells, and not in CRPC-NE. HOXB13 is a homeobox transcription factor involved in prostate development and displays context-dependent roles in PCa: it can act as a collaborator or a negative regulator of AR signaling ${ }^{43,81}$, it cooperates with the AR-V7 splice variant found in a subset of CRPC-Adeno ${ }^{82}$, and germline gain-of-function G84E HOXB13 mutations are associated with increased prostate cancer risk ${ }^{83}$. The fact that by ChIP-seq, SWI/SNF colocalizes with HOXB13 at active chromatin sites in prostatic adenocarcinoma cells, further supports the hypothesis that interaction between SWI/SNF and lineage-specific factors in PCa may be meaningful at the functional level.

In conclusion, this work confirms that SWI/SNF has tumorpromoting functions in $\mathrm{PCa}$, including the lethal CRPC-NE. Our findings provide a rationale to further study selected SWI/SNF subunits as potential therapeutic targets in PCa.

\section{Methods}

Genomic analysis. Matched tumor and normal WES data of localized and advanced prostate cancer from The Cancer Genome Atlas ${ }^{84}$, SU2C-PCF ${ }^{5}$ and from the Weill Cornell Medicine cohort ${ }^{4}$ were uniformly analyzed for somatic copy number aberrations (SCNA) with $\mathrm{CNVkit}^{85}$, and for single nucleotide variations (SNVs) and indels with MuTect2 ${ }^{86}$. SNVs and Indels were annotated with variant effect predictor (VEP) ${ }^{87}$ and only mutations with HIGH or MODERATE predicted impact on a transcript or protein (https://www.ensembl.org/info/genome/ variation/prediction/predicted data.html) were retained. All samples with tumor ploidy and purity estimated using CLONET $^{88}$ were retained in the analyses and processed for allele specific characterization. The integrated dataset includes 299 


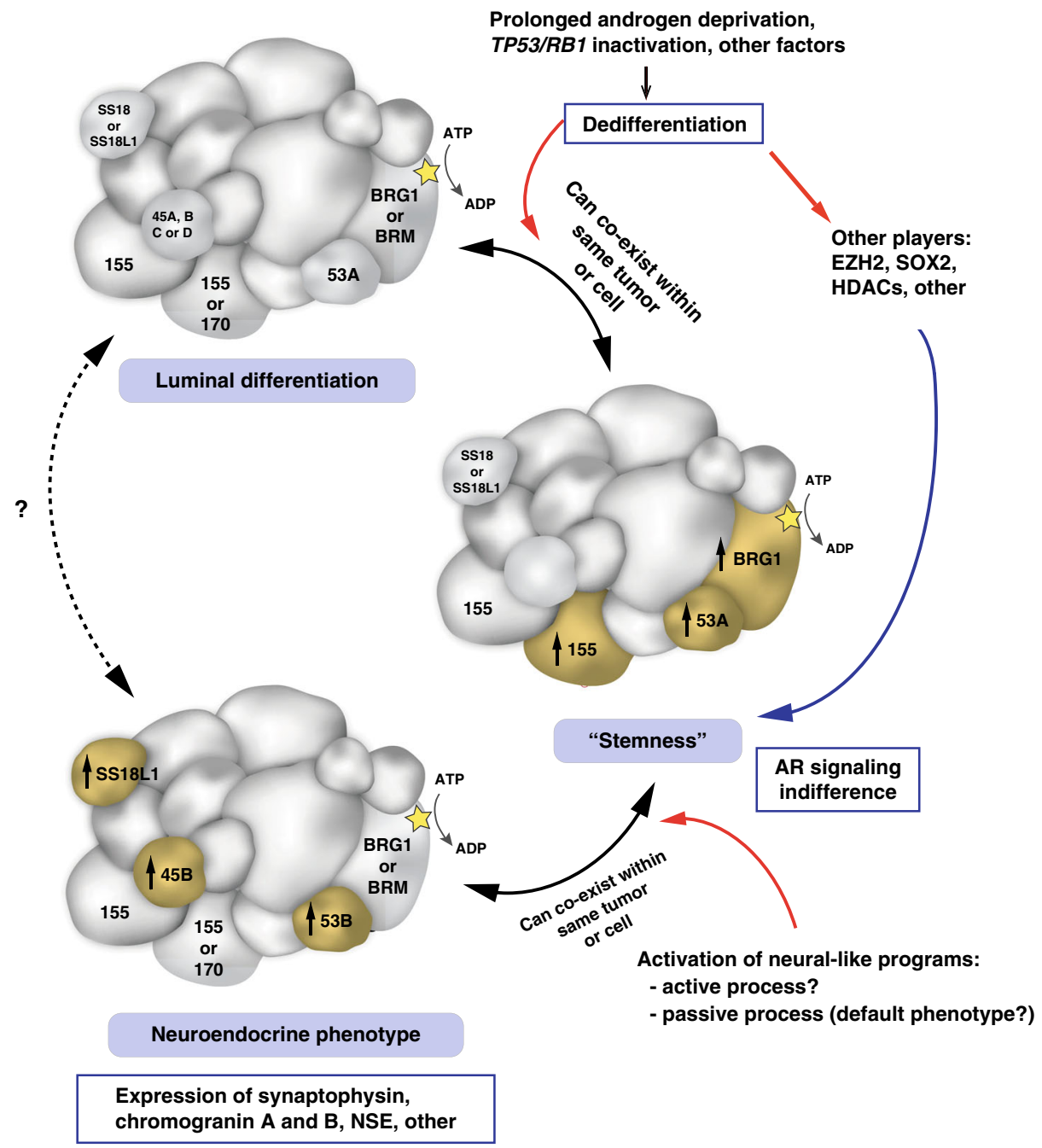

Fig. 5 Schematic representation of putative specialized SWI/SNF assemblies in prostate cancer cells. Hypothetical SWI/SNF assemblies are shown in the context of current knowledge about prostate cancer phenotype plasticity. Subunits of particular interest are annotated with their names. Two names within a subunit indicate possible incorporation of either one of the two paralogs. Subunit sizes are approximately indicative of their molecular weights. 155: BAF155, 170: BAF170, 53A: BAF53A, 53B: BAF53B, 45B: BAF45B, AR: Androgen receptor.

unique hormone treatment-naïve prostatic adenocarcinoma (Adeno), 245 castration resistant prostate adenocarcinoma (CRPC-Adeno), and 56 castration resistant neuroendocrine prostate carcinoma (CRPC-NE) patients. Two-tailed proportion test has been used to check enrichment of hemizygous deletion and copy number neutral loss.

RNA-seq data analysis of human samples. RNA-seq data from 32 normal prostate samples $89,90,400$ localized PCa ${ }^{37,89,90}$ and 120 CRPC-Adenos and 20 CRPC-NE patients ${ }^{4,5}$ were utilized for the initial investigation of the SWI-SNF complex units levels and were processed as follows. Reads (FASTQ files) were mapped to the human genome reference sequence (hg19/GRC37) using STAR v2.3.0 $\mathrm{e}^{91}$, and the resulting alignment files were converted into Mapped Read Format (MRF) for gene expression quantification using RSEQtools ${ }^{92}$ and GENCODE v19 (http://www.gencodegenes.org/releases/19.html) as reference gene annotation set. A composite model of genes based on the union of all exonic regions from all gene transcripts was used, resulting in a set of 20,345 proteincoding genes. Normalized expression levels were estimated as FPKM. After converting the FPKM via $\log 2(\mathrm{FPKM}+1)$, differential expression analysis was performed using Mann-Whitney Wilcoxon test. RNA-seq data of the SU2C-PCF cohort were downloaded from original study ${ }^{5}$. NEPC score and AR signaling score were inferred as previously described ${ }^{5}$. Gleason scores of the TCGA PCas were retrieved from the original study ${ }^{37}$. RNA-seq data and Gleason score from the TCGA PCa dataset were retrieved from the TCGA data portal using TCGAbiolinks $\mathrm{R}$ package $\mathrm{v} 2.12 .2^{93}$.

Immunohistochemistry. Immunohistochemistry (IHC) was performed on sections of formalin-fixed paraffin-embedded patient tissue (FFPE) using a Bond III automated immunostainer and the Bond Polymer Refine detection system (Leica Microsystems, IL, USA). Slides were de-paraffinized and heat-mediated antigen retrieval using the Bond Epitope Retrieval 1 solution at pH6 (H1) or Bond Epitope Retrieval 2 solution at $\mathrm{pH} 9$ (H2) or enzyme-mediated antigen retrieval (E1) was performed. All antibodies, dilutions and conditions used are listed in Supplementary Table 3 .

The intensity of nuclear immunostaining for SWI/SNF subunits was evaluated on tissue micro-arrays (TMAs) and whole slide sections by a pathologist (J.C.) blinded to additional pathological and clinical data, and was scored as negative (score 0 ), weak (score 1), moderate (score 2), or strong (score 3). Association between disease state and staining intensity (negative/weak vs. moderate/strong) was examined using the two-tailed Fisher's exact test.

Analysis of SMARCA4 and SMARCA2 expression in localized PCa vs. clinical outcome. The patient cohort with localized PCa and available clinical and follow-up information has been previously described ${ }^{30}$. IHC for SMARCA4 and SMARCA2 was performed on TMAs constructed from these patients' prostatectomy specimens. Staining intensity was scored by a pathologist (J.C.) blinded to the clinical data, using the digital online TMA scoring tool Scorenado (University of Bern, Switzerland). The Kaplan-Meier method was used to estimate patients' overall survival. The association between SMARCA4 and SMARCA2 expression (strong vs. moderate/weak/negative) and overall survival was examined using the log-rank test and multivariable Cox proportional hazards regression models. Ninety-five percent confidence intervals were calculated to assess the precision of the obtained hazard ratios. All $p$-values were two-sided, and statistical significance was evaluated at the 0.05 alpha level. All analyses were performed in R (3.5.1) for Windows. 
Development of a SMARCA4 knock-down signature. We defined the SMARCA4 knock-down signature by selecting a list of differentially expressed genes between SMARCA4 siRNA-mediated knock-down and Scrambled control in the LNCaP cell line with a log fold change of 1.5 and an FDR $<0.01$. For each sample, gene expression data were first normalized by $z$-score transformation. Then signature score was calculated as a weighted sum of normalized expression of the genes in the signature and was finally re-scaled with the $2.5 \%$ and $97.5 \%$ quantiles equaled -1 and +1 , respectively. We defined samples with low SMARCA4 knock-down signature score as the $25 \%$ of cases with the lowest scores, and samples with high signature score as the $25 \%$ of cases with the highest scores.

\section{Validation of SMARCA4 knock-down signature in multiple clinical cohorts.} SMARCA4 knock-down generated signature was applied to two CRPC cohorts consisting of 332 patients from the Stand Up To Cancer-Prostate Cancer Foundation (SU2C-PCF) trial treated with ARSi (recently published by Abida et al. ${ }^{5}$ ) and 47 patients from the Weil Cornell Medicine (WCM) cohort (published by Beltran et $\mathrm{al}^{4}$ ) and on one cohort of localized, hormone treatment-naïve PCa consisting of 495 patients from The Cancer Genome Atlas (TCGA).

Results from the signature was then correlated with NEPC score and AR signaling scores for the SU2C-PCF and the WCM dataset and with Gleason score for the TCGA dataset.

Decipher GRID analysis. For prospective Decipher GRID and JHMI cohort, tumor RNA was extracted from FFPE blocks or slides after macrodissection guided by a histologic review of the tumor lesion by a GU pathologist. RNA extraction and microarray hybridization were all done in a Clinical Laboratory Improvement Amendments (CLIA)-certified laboratory facility (GenomeDx Biosciences, San Diego, CA, USA). Total RNA was amplified and hybridized to Human Exon 1.0 ST GeneChips (Thermo-Fisher, Carlsbad, CA). All data was normalized using the Single Channel Array Normalization (SCAN) algorithm ${ }^{94}$. Decipher scores were calculated based on the predefined 22-markers ${ }^{38}$. Patients with high Decipher $(>0.7)$ were categorized as genomically high risk patients. Mann-Whitney U test was used to assess score differences across Gleason score groups and Mann-Kendall trend test was used to test the association between the percentage of high Decipher scores across deciles of the SMARCA4 knock-down signature. Kaplan-Meier analysis and Cox proportional hazard model was used to associate SMARCA4 knock-down signature with time to metastasis in the JHMI cohort.

Cell culture. Commercially available PCa cell lines (RWPE-1, LNCaP, 22Rv1, VCaP, LAPC4, PC3, DU145, NCI-H660, C4-2) were purchased from ATCC and maintained according to ATCC protocols. WCM154 and WCM155 CRPC-NE cell lines have been previously established and were maintained in two-dimensional monolayer culture according to the previously described protocol ${ }^{29}$. LNCaP-AR cells were a kind gift from Dr. Sawyers and Dr. Mu (Memorial Sloan Kettering Cancer Center) and were cultured as previously described ${ }^{12}$. MSKCC-PCa3 CRPCAdeno cells were a kind gift from Dr. Chen (Memorial Sloan Kettering Cancer Center) and were maintained identically to WCM154 and WCM155 cells. All cell lines used and their phenotype are listed in Supplementary Table 4. Cell cultures were regularly tested for Mycoplasma contamination and confirmed to be negative.

Cell transfection and siRNA-mediated knock-down. ON-TARGET plus siRNA SMARTpool siRNAs against SMARCA4, SMARCA2, SMARCC1, SMARCC2, and REST were purchased from Dharmacon. Transfection was performed overnight on attached cells growing in 6-well plates using the Lipofectamine 3000 reagent (Thermo Fisher Scientific) to the proportions of $10 \mu \mathrm{L}$ of $20 \mu \mathrm{M}$ siRNA per well. Cells were harvested for protein and RNA extraction $72 \mathrm{~h}$ after transfection.

Cell infection, shRNA-mediated knock-down and gene overexpression. The $A C T L 6 B$ shRNA and the matching Scrambled shRNA control were a kind gift from Dr. Cigall Kadoch (Dana Farber Cancer Institute). The vector was pGIPZ and the target sequence was: sh\#1-TGGATCACACCTACAGCAA. The DPF1 shRNA and the corresponding Scrambled shRNA control were purchased from Genecopoeia. The vector was psi-LVRU6GP and the target sequences were: sh\#1-GAATTAACT TGTTCTGTGTAT, Scrambled control-GCTTCGCGCCGTAGTCTTA. For infection, WCM155 cells were collected, resuspended in media containing Polybrene (Millipore) and lentiviral particles, and centrifuged at $800 \times g$ at room temperature for $60 \mathrm{~min}$. Both vectors included a GFP reporter and infection efficiency was confirmed by green fluorescence. Cells were harvested for protein and RNA extraction $72 \mathrm{~h}$ after transfection. Given the short-term nature of the experiments, selection was not performed. For the SMARCA4 or SMARCA2 overexpression experiment, lentiviral particles were prepared as described above using the pEZ-Lv203 vector (SMARCA4 gene, eGFP reporter), the pEZLv216 vector (SMARCA2 gene, mCherry reporter) (all vectors Genecopoeia, MD, USA; all sequence-verified). 22Rv1 cells were infected as described above, cultured and sorted based on the expression of the fluorescent reporter.

Immunoblotting. Cells were lysed in RIPA buffer with protease and phosphatase inhibitors (Thermo Fisher Scientific) and total protein concentration was measured using the DC Protein Assay (Bio-Rad). Protein samples were resolved in SDSPAGE, transferred onto a nitrocellulose membrane using the iBlot 2 dry blotting system (Thermo Fisher Scientific) and incubated overnight at $4{ }^{\circ} \mathrm{C}$ with primary antibodies dissolved in 5\% Blotting-Grade Blocker (Bio-Rad). All primary antibodies and dilutions used are listed in Supplementary Table 3. After 3 washes, the membrane was incubated with secondary antibody conjugated to horseradish peroxidase for $1 \mathrm{~h}$ at room temperature. After 3 washes, signal was visualized by chemiluminescence using the Luminata Forte substrate (Thermo Fisher Scientific) and images were acquired with the ChemiDoc ${ }^{\text {tx }}$ Touch Imaging System (Bio-Rad, Hercules, CA). When blotting of a single membrane for different proteins was necessary, the membrane was stripped using the Restore PLUS Stripping Buffer (Thermo Fisher Scientific) according to producer's instructions and the immunoblotting process was repeated.

RNA extraction from cells, RNA sequencing and analysis, qPCR. Total RNA was extracted from cells using the Maxwell 16 LEV simplyRNA Purification Kit and the Maxwell 16 Instrument. RNA integrity was verified using the Agilent Bioanalyzer 2100 (Agilent Technologies). cDNA was synthesized from total RNA using Superscript III (Invitrogen). Library preparation was performed using TruSeq RNA Library Preparation Kit v2. RNA sequencing was performed on the HiSeq 2500 sequencer to generate $2 \times 75$ bp paired-end reads.

Sequence reads were aligned using STAR two-pass ${ }^{95}$ to the human reference genome GRCh37. Gene counts were quantified using the "GeneCounts" option. Per-gene counts-per-million (CPM) were computed and $\log _{2}$-transformed adding a pseudo-count of 1 to avoid transforming 0 . Genes with $\log _{2}$-CPM $<1$ in more than three samples were removed. Unsupervised clustering was performed using the top 500 most variable genes, Euclidean distance as the distance metric and the Ward clustering algorithm. When required, the batch effect was removed using the function removeBatchEffect from the limma R package for data visualization. For differential expression the batch factor was included in the design matrix.

Differential expression analysis between knock-down cells and control samples was performed using the edgeR v3.28.1 package ${ }^{96}$. Normalization was performed using the "TMM" (weighted trimmed mean) method and differential expression was assessed using the quasi-likelihood F-test.

Genes with FDR $<0.05$ and $>2$-fold were considered significantly differentially expressed.

Gene Set Enrichment Analysis (GSEA) was performed using the Preranked tool $^{97}$ for $\mathrm{C} 2$ (canonical pathways) and $\mathrm{H}$ (hallmark gene sets) ${ }^{98}$. Genes were ranked based on the T-statistic from the differential expression analysis.

Primer sequences used for qPCR are available in Supplementary Table 5.

Cell growth experiments. Cells were treated with siRNA ( 3 pmol) against SMARCA4, SMARCA2, SMARCC1, SMARCC2 or with a scrambled control for $24 \mathrm{~h}$. LNCaP and C4-2 cells were then seeded in Poly-L-Lysine coated 96-well plates (2000 cells/well) and WCM154 cells were seeded in a collagen-coated 96-well plates (5000 cells/well). Cell viability was determined after $24,48,72$, and $96 \mathrm{~h}$ with a Tecan Infinite M200PRO reader using the CellTiter-Glo ${ }^{\oplus}$ Luminescent Cell Viability Assay according to manufacturer's directions (Promega). Cell confluence was determined using the Incucyte S3 instrument and the IncuCyte S3 2018B software (Essen Bioscience, Germany). Values were calculated as $x$-fold of cells transfected with siRNA for $0 \mathrm{~h}$.

Co-immunoprecipitation and mass spectrometry analysis. For the coimmunoprecipitation (co-IP) using an anti-BAF155 antibody, nuclear fractions of LNCaP-AR and NCI-H660 cells were isolated using the using the Universal CoIP Kit (Actif Motif). Chromatin of the nuclear fraction was mechanically sheared using a Dounce homogenizer. Nuclear membrane and debris were pelleted by centrifugation and protein concentration of the cleared lysate was determined with the Pierce BCA Protein Assay Kit (Thermo Fisher Scientific). $2 \mu \mathrm{g}$ of the antiBAF155 antibody (ab172638, Abcam) and $2 \mu \mathrm{g}$ of rabbit IgG Isotype Control antibody (026102, Thermo Fisher Scientific) were incubated with $2 \mathrm{mg}$ protein supernatant overnight at $4{ }^{\circ} \mathrm{C}$ with gentle rotation. The following morning, $30 \mu \mathrm{l}$ of Protein G Magnetic Beads (Active Motif) were washed twice with $500 \mu \mathrm{l}$ CoIP buffer and incubated with Antibody-containing lysate for $1 \mathrm{~h}$ at $4{ }^{\circ} \mathrm{C}$ with gentle rotation. Bead-bound SWI/SNF complexes were washed 3 times with CoIP buffer and twice with a buffer containing $150 \mathrm{mM} \mathrm{NaCl}, 50 \mathrm{mM}$ Tris-HCL (pH 8) and Protease and Phosphatase inhibitors. Air-dried and frozen $\left(-20^{\circ} \mathrm{C}\right)$ beads were subjected to mass spectrometry (MS) analysis. Proteins on the affinity pulldown beads were re-suspended in $8 \mathrm{M}$ Urea $/ 50 \mathrm{mM}$ Tris- $\mathrm{HCl} \mathrm{pH} 8$, reduced $30 \mathrm{~min}$ at $37^{\circ} \mathrm{C}$ with DTT $0.1 \mathrm{M} / 100 \mathrm{mM}$ Tris- $\mathrm{HCl} \mathrm{pH} 8$, alkylated $30 \mathrm{~min}$ at $37^{\circ} \mathrm{C}$ in the dark with IAA $0.5 \mathrm{M} / 100 \mathrm{mM}$ Tris- $\mathrm{HCl} \mathrm{pH} 8$, diluted with 4 volumes of $20 \mathrm{mM}$ Tris- $\mathrm{HCl} \mathrm{pH} 8 / 2 \mathrm{mM} \mathrm{CaCl}_{2}$ prior to overnight digestion at room temperature with $100 \mathrm{ng}$ sequencing grade trypsin (Promega). Samples were centrifuged and the magnetic beads trapped by a magnet holder in order to extract the peptides in the supernatant.

The digests were analyzed by liquid chromatography (LC)-MS/MS (PROXEON coupled to a QExactive HF mass spectrometer, ThermoFisher Scientific) with three injections of $5 \mu$ digests. Peptides were trapped on a $\mu$ Precolumn C18 PepMap100 ( $5 \mu \mathrm{m}, 100 \AA, 300 \mu \mathrm{m} \times 5 \mathrm{~mm}$, ThermoFisher Scientific, Reinach, Switzerland) and 
separated by backflush on a C18 column $(5 \mu \mathrm{m}, 100 \AA, 75 \mu \mathrm{m} \times 15 \mathrm{~cm}, \mathrm{C} 18)$ by applying a 60 -min gradient of $5 \%$ acetonitrile to $40 \%$ in water, $0.1 \%$ formic acid, at a flow rate of $350 \mathrm{nl} / \mathrm{min}$. The Full Scan method was set with resolution at 60,000 with an automatic gain control (AGC) target of 1E06 and maximum ion injection time of $50 \mathrm{~ms}$. The data-dependent method for precursor ion fragmentation was applied with the following settings: resolution 15,000, AGC of 1E05, maximum ion time of $110 \mathrm{~ms}$, mass window $1.6 \mathrm{~m} / \mathrm{z}$, collision energy 28 , under fill ratio $1 \%$, charge exclusion of unassigned and $1+$ ions, and peptide match preferred, respectively.

MS data was interpreted with MaxQuant (version 1.6.1.0) against a SwissProt human database (release 2019_02) using the default MaxQuant settings, allowed mass deviation for precursor ions of $10 \mathrm{ppm}$ for the first search, maximum peptide mass of $5500 \mathrm{Da}$, match between runs activated with a matching time window of $0.7 \mathrm{~min}$ and the use of non-consecutive fractions for the different pulldowns to prevent over-fitting. Settings that differed from the default setting included: strict trypsin cleavage rule allowing for 3 missed cleavages, fixed carbamidomethylation of cysteines, variable oxidation of methionines and acetylation of protein $\mathrm{N}$ termini.

Protein intensities are reported as MaxQuant's Label Free Quantification (LFQ) values, as well as Top3 values (sum of the intensities of the three most intense peptides); for the latter, variance stabilization was used for the peptide normalization, and missing peptide intensities were imputed in the following manner: if there was at least two evidences in one group of replicates, the missing value was drawn from a Gaussian distribution of width 0.3 centered at the sample distribution mean minus $1.8 \times$ the sample standard deviation. Imputation at protein level for both LFQ and Top3 values was performed if there were at least two measured intensities in at least one group of replicates; missing values in this case were drawn from a Gaussian distribution of width 0.2 centered at the sample distribution mean minus $2.5 \mathrm{x}$ the sample standard deviation. Differential expression tests were performed using the moderated $t$-test empirical Bayes $(\mathrm{R}$ function EBayes from the limma package version 3.40.6) on imputed LFQ and Top3 protein intensities. The Benjamini and Hochberg method was further applied to correct for multiple testing. The criterion for statistically significant differential expression is that the maximum adjusted $p$-value for large fold changes is 0.05 , and that this maximum decreases asymptotically to 0 as the log2 fold change of 1 is approached (with a curve parameter of one time the overall standard deviation).

Please see below, the description of the methods for the second Co-IP (validation experiment) using an anti-BRG1 antibody in LNCaP and NCIH660 cells.

CRISPR-Cas9 mediated TP53 and RB1 knock-out. To generate the stable p53 and RB1 knockout cells, all-in-one CRISPR plasmids with mCherry reporter were purchased from Genecopoeia (Cat \# HCP218175-CG01, HCP216131-CG01). Cells were transfected with CRISPR plasmids, selected with puromycin and sorted for mCherry positivity. TP53 gRNA sequences used: TCGACGCTAGGATCTGACTG, CGTCGAGCCCCCTCTGAGTC, CCATTGTTCAATATCGTCCG. RB1 gRNA sequences used: CGGTGGCGGCCGTTTTTCGG, CGGTGCCGGGGGTTCCGC GG, CGGAGGACCTGCCTCTCGTC. Control gRNA sequence: GGCTTCGCGCC GTAGTCTTA.

Single cell RNA-sequencing (scRNAseq). scRNAseq was performed for two CRPC-NE organoids in 3D culture: MSK PCa1 and MSK PCa16. Cell counting and viability tests were conducted using a Moxi Go II Flow Cytometer (Orflo Technologies) with trypan blue and Propidium Iodide staining. Subsequently, GEM generation and barcoding, reverse transcription, cDNA amplification and $3^{\prime}$ Gene Expression library generation steps were all performed according to the Chromium Single Cell 3' Reagent Kits v3 user Guide (10× Genomics CG000183 Rev B). Specifically, 32.0, 11.4, and $40.0 \mu \mathrm{L}$ of PCa1, PCa8, and PCa16 cell suspension (100, 750 , and 200 cells $/ \mu \mathrm{L}$ ) were used for a targeted cell recovery of 2000, 5000, and 2000 cells, respectively. GEM generation was followed by a GEM-reverse transcription incubation, a clean-up step and 12 cycles of cDNA amplification. The resulting cDNA was assessed for quantity and quality using fluorometry and capillary electrophoresis, respectively. The cDNA libraries were pooled and sequenced paired-end and single indexed on an illumina NovaSeq 6000 sequencer with a shared NovaSeq 6000 S2 Reagent Kit (100 cycles). The read-set up was as follows: read $1=28$ cycles, i7 index $=8$ cycles, $i 5=0$ cycles and read $2=91$ cycles. An average of 300,753,777 reads/library were obtained, equating to an average of 111, $978 \mathrm{reads} / \mathrm{cell}$. All steps were performed at the Next Generation Sequencing Platform, University of Bern. Data demultiplexing was performed using SEURAT v. 3.1.5 package (PMID 29608179). Low quality cells and multiplets were excluded by removing cells with unique feature counts over 5500 or less than 1000. Cells containing mitochondrial gene counts greater than $25 \%$ were also removed. Data were then scaled to 10,000 and $\log$ transformed. Only cells expressing SOX2 and SMARCA4 genes were included. Boxplots were drawn using GGPLOT2 3.3.0 (https://ggplot2.tidyverse.org) and $p$-values were calculated using Wilcoxon test.

ChIP-sequencing data analysis. ChIP-seq peaks for SMARCC1 and HOXB13 in LNCaP cells were downloaded from GEO: GSE110655 and GSE94682, respectively. ChIP-seq peaks for H3K27ac and H3K27me3 in LNCaP cells were from data published by Sandoval et al. ${ }^{71}$. Peak comparison was performed using BEDTOOLS v2-29.0 (https://bedtools.readthedocs.io/en/latest/\#).

Co-immunoprecipitation using the anti-SMARCA4 antibody and mass spectrometry analysis. For the second Co-IP (validation experiment) using an antiSMARCA4 antibody (results shown in Supplementary Fig. 26 and Supplementary Data 9), SWI/SNF complexes were isolated from the nuclear fraction of LNCaP (adenocarcinoma) or NCI-H660 (CRPC-NE) cells, which was prepared using the Universal CoIP Kit (Active Motif). Briefly, anti-Brg-1 antibodies (H-10, Santa Cruz Biotechnology) were cross-linked using Dimethyl pimelimidate dihydrochloride (Sigma-Aldrich) to Protein G conjugated magnetic beads (Bio-Rad). $30 \mu \mathrm{g}$ of crosslinked antibodies were incubated with $0.8-1 \mathrm{mg}$ of nuclear lysates overnight. Beadbound BAF complexes were washed and eluted using $8 \mathrm{M}$ urea buffer. The obtained protein complexes were subjected to immunoblotting and MS analysis.

For MS analysis, the eluted proteins were precipitated with trichloroacetic acid (TCA, 20\% w/v), rinsed three times with acetone, and dried at room temperature. The pellets were re-suspended in $50 \mu \mathrm{L}$ resuspension buffer $(8 \mathrm{M}$ urea, $50 \mathrm{mM}$ ammonium bicarbonate, and $5 \mathrm{mM}$ DTT) and subjected to reduction and alkylation by adding $15 \mathrm{mM}$ iodoacetamide to each sample for $30 \mathrm{~min}$ in the dark at room temperature, followed by addition of $5 \mathrm{mM}$ DTT to quench the reaction. Samples were diluted to a final concentration of $2 \mathrm{M}$ urea and digested with LysC at room temperature overnight, and then diluted further to $1 \mathrm{M}$ urea and digested with Trypsin at $37^{\circ} \mathrm{C}$ overnight (for each enzyme a ratio of 1:125 enzyme:protein was used).

Samples were labeled using reductive dimethylation. Labeling was done while the peptides were bound to the solid phase C18 resin in self-packed STAGE Tip micro-columns ${ }^{99}$. Stage tips were washed with methanol, acetonitrile (ACN) $70 \%$ $\mathrm{v} / \mathrm{v}$ and formic acid (FA) $1 \% \mathrm{v} / \mathrm{v}$. Samples were acidified by adding $100 \%$ FA to a final concentration of $2 \%$ FA before loading. After sample loading, stage tips were washed with $1 \% \mathrm{FA}$ and phosphate/citrate buffer $(0.23 \mathrm{M}$ sodium phosphate and $86.4 \mathrm{mM}$ citric acid [pH 5.5]). At this point, the "light" solution $\left(0.4 \% \mathrm{CH}_{2} \mathrm{O}\right.$ and $\left.60 \mathrm{mM} \mathrm{NaBH}{ }_{3} \mathrm{CN}\right)$, or "heavy" solution $\left(0.4 \% \mathrm{CD}_{2} \mathrm{O}\right.$ and $\left.60 \mathrm{mM} \mathrm{NaBD}{ }_{3} \mathrm{CN}\right)$ was added twice on each stage tip to label the peptides. A final wash with $1 \%$ FA was performed prior to elution with $70 \% \mathrm{ACN}$ and $1 \% \mathrm{FA}$. Samples were dried under vacuum, resuspended in 5\% FA, and mixed together in equal amounts for analysis using an Orbitrap Fusion Mass Spectrometer. Peptides were introduced into the mass spectrometer by nano-electrospray as they eluted off a self-packed $40 \mathrm{~cm}, 75 \mu \mathrm{m}$ (ID) reverse-phase column packed with $1.8 \mu \mathrm{m}, 120 \AA$ pore size, SEPAX C18 resin. Peptides were separated with a gradient of 5-25\% buffer B $(99.9 \% \mathrm{ACN}, 0.1 \% \mathrm{FA})$ with a flow rate of $350 \mathrm{nl} / \mathrm{min}$ for $65 \mathrm{~min}$. For each scan cycle, one high mass resolution full MS scan was acquired in the Orbitrap mass analyzer at a resolution of $120 \mathrm{~K}$, AGC value of 500,000 , in a $\mathrm{m} / \mathrm{z}$ scan range of $375-1400$, max acquisition time of $100 \mathrm{~ms}$ and up to 20 parent ions were chosen based on their intensity for collision induced dissociation (normalized collision energy $=35 \%$ ) and MS/MS fragment ion scans at low mass resolution in the linear ion trap. Dynamic exclusion was enabled to exclude ions that had already been selected for MS/MS in the previous $40 \mathrm{~s}$. Ions with a charge of +1 and those whose charge state could not be assigned were also excluded. All scans were collected in centroid mode. Two biological replicates for each condition were processed and analyzed.

MS2 spectra were searched using SEQUEST (version 28 revision 13) against a composite database containing all Swiss-Prot reviewed human protein sequences (20,193 target sequences, downloaded from www.uniprot.org March 18, 2016) and their reversed complement, using the following parameters: a precursor mass tolerance of $\pm 25 \mathrm{ppm} ; 1.0$ Da product ion mass tolerance; tryptic digestion up to two missed cleavages; static modifications of carbamidomethylation on cysteine $(+57.0214)$ and dimethylation on $n$-termini and lysines $(+28.0313)$; dynamic modifications of methionine oxidation $(+15.9949)$ and heavy dimethylation on $\mathrm{N}$-termini and lysines $(+6.03766)$. Peptide spectral matches (PSMs) were filtered to $1 \%$ FDR using the target-decoy strategy ${ }^{100}$ combined with linear discriminant analysis (LDA) ${ }^{101}$ using several different parameters including Xcorr, $\Delta \mathrm{Cn}^{\prime}$, precursor mass error, observed ion charge state, and predicted solution charge state. Linear discriminant models were calculated for each LC-MS/MS run using peptide matches to forward and reversed protein sequences as positive and negative training data. PSMs within each run were sorted in descending order by discriminant score and filtered to a $1 \%$ FDR as revealed by the number of decoy sequences remaining in the data set. The data were further filtered to control protein level FDRs. Peptides were combined and assembled into proteins. Protein scores were derived from the product of all LDA peptide probabilities, sorted by rank, and filtered to $1 \%$ FDR as described for peptides. The FDR of the remaining peptides fell dramatically after protein filtering. The data were further filtered to require a minimum of 8 PSMs per protein. All peptides were required to have a sum of heavy and light signal-tonoise $(\mathrm{SN}) \geq 10$. Protein ratios were calculated as the $\log _{2}$ ratio of the total $\mathrm{SN}$ of all experimental sample peptide values over that for $\mathrm{IgG}$ control sample peptides. For a small number of the most highly enriched proteins, the control value was zero (this is the theoretical ideal). In these cases, we imputed a value of one for ratio calculations. Subsequent visualization and statistical analysis was done with Perseus and $\mathrm{R}$ program ${ }^{102}$ 
SMARCC1 Co-IP immunoblotting. For the co-immunoprecipitation (co-IP), using an anti-BAF155 antibody, nuclear fractions of LNCaP-AR and NCI-H660 cells were isolated using the using the Universal CoIP Kit (Active Motif). Chromatin of the nuclear fraction was mechanically sheared using a Dounce homogenizer. Nuclear membrane and debris were pelleted by centrifugation and protein concentration of the cleared lysate was determined with the Pierce BCA Protein Assay Kit (Thermo Fisher Scientific). One microgram of the rabbit anti-BAF155 antibody (ab172638, Abcam) and $1 \mu \mathrm{g}$ of rabbit IgG Isotype Control antibody (026102, Thermo Fisher Scientific) were incubated with $1 \mathrm{mg}$ protein supernatant overnight at $4{ }^{\circ} \mathrm{C}$ with gentle rotation. The following morning, $30 \mu$ of Protein G Magnetic Beads (Active Motif) were washed twice with $500 \mu \mathrm{l}$ CoIP buffer and incubated with Antibody-containing lysate for $2 \mathrm{~h}$ at $4{ }^{\circ} \mathrm{C}$ with gentle rotation. Bead-bound SWI/SNF complexes were washed twice with CoIP buffer and twice with a buffer containing $150 \mathrm{mM} \mathrm{NaCl}, 50 \mathrm{mM}$ Tris- $\mathrm{HCL}(\mathrm{pH}$ 8) and Protease and Phosphatase inhibitors. Washing procedure was executed at $4{ }^{\circ} \mathrm{C}$ with gentle rotation. Beadbound protein and Input controls are reduced and denatured in $40 \mu \mathrm{l} \mathrm{Laemmli}$ buffer containing DTT through boiling for $5 \mathrm{~min}$ at $95^{\circ} \mathrm{C}$. Magnetic beads are removed from solution and $20 \mu \mathrm{l}$ of reduce protein is loaded on an SDS-PAGE gel with subsequent immunoblotting using iBlot (Life Technologies). Membranes were blocked in 5\% dry-milk solution and then incubated over night with respective antibodies against targets of interest. Protein signal was detected using HRP-labeled native anti-rabbit IgG antibody (CST, \#5127) and ECL substrate solution (Merck Millipore) using the Fusion FX.

RNA isolation and qPCR. Cells were first seeded in $10 \mathrm{~cm}$-petridish and grown until they reached a confluency of approx. $90 \%$. The cells were then harvested for RNA isolation using the RNeasy Mini Kit (Qiagen). Synthesis of complementary DNAs (cDNAs) using FIREScript RT cDNA Synthesis Kit (Solis BioDyne) and real-time reverse transcription PCR (RT-PCR) assays using HOT FIREPol EvaGreen qPCR Mix Plus (Solis BioDyne) were performed using and applying the manufacturer protocols. Relative mRNA levels of each gene shown were normalized to the expression of the average of housekeeping genes GAPDH and ACTB. The sequences of the primers for qRT-PCR assays can be found in Supplementary Table 5 .

Reporting summary. Further information on research design is available in the Nature Research Reporting Summary linked to this article.

\section{Data availability}

Data generated during this study have been submitted on the European Genomephenome Archive under the accession EGAS00001004177 (https://ega-archive.org/ datasets/EGAD00001005800). The mass spectrometry proteomics data that support the findings of this study have been deposited to the ProteomeXchange Consortium (http:// proteomecentral.proteomexchange.org) via the PRIDE partner repository with the dataset identifier PXD016861. Source data are provided with this paper.

Received: 8 February 2020; Accepted: 7 October 2020;

Published online: 03 November 2020

\section{References}

1. Bray, F. et al. Global cancer statistics 2018: GLOBOCAN estimates of incidence and mortality worldwide for 36 cancers in 185 countries. CA Cancer J. Clin. 68, 394-424 (2018).

2. Rawla, P. Epidemiology of prostate cancer. World J. Oncol. 10, 63-89 (2019).

3. Robinson, D. et al. Integrative clinical genomics of advanced prostate. Cancer Cell 162, 454 (2015).

4. Beltran, H. et al. Divergent clonal evolution of castration-resistant neuroendocrine prostate cancer. Nat. Med. 22, 298-305 (2016).

5. Abida, W. et al. Genomic correlates of clinical outcome in advanced prostate cancer. Proc. Natl Acad. Sci. USA 116, 11428-11436 (2019).

6. Epstein, J. I. et al. Proposed morphologic classification of prostate cancer with neuroendocrine differentiation. Am. J. Surg. Pathol. 38, 756-767 (2014).

7. Rickman, D. S., Beltran, H., Demichelis, F. \& Rubin, M. A. Biology and evolution of poorly differentiated neuroendocrine tumors. Nat. Med. 23, 1-10 (2017).

8. Aggarwal, R. et al. Clinical and genomic characterization of treatmentemergent small-cell neuroendocrine prostate cancer: a multi-institutional prospective study. J. Clin. Oncol. 36, 2492-2503 (2018)

9. Bluemn, E. G. et al. Androgen receptor pathway-independent prostate cancer is sustained through FGF signaling. Cancer Cell 32, 474-489 e6 (2017).

10. Metzger, A. L. et al. Patterns of care and outcomes in small cell carcinoma of the prostate: a national cancer database analysis. Prostate 79, 1457-1461 (2019).

11. $\mathrm{Ku}, \mathrm{S}$. Y. et al. Rb1 and Trp53 cooperate to suppress prostate cancer lineage plasticity, metastasis, and antiandrogen resistance. Science 355, 78-83 (2017).
12. $\mathrm{Mu}, \mathrm{P}$. et al. SOX 2 promotes lineage plasticity and antiandrogen resistance in TP53- and RB1-deficient prostate cancer. Science 355, 84-88 (2017).

13. Davies, A. H., Beltran, H. \& Zoubeidi, A. Cellular plasticity and the neuroendocrine phenotype in prostate cancer. Nat. Rev. Urol. 15, 271-286 (2018).

14. Kadoch, C. et al. Proteomic and bioinformatic analysis of mammalian SWI/ SNF complexes identifies extensive roles in human malignancy. Nat. Genet. 45, 592-601 (2013).

15. Shain, A. H. \& Pollack, J. R. The spectrum of SWI/SNF mutations, ubiquitous in human cancers. PLoS ONE 8, e55119 (2013).

16. Kadoch, C. \& Crabtree, G. R. Mammalian SWI/SNF chromatin remodeling complexes and cancer: mechanistic insights gained from human genomics. Sci. Adv. 1, e1500447 (2015).

17. Hargreaves, D. C. \& Crabtree, G. R. ATP-dependent chromatin remodeling: genetics, genomics and mechanisms. Cell Res. 21, 396-420 (2011).

18. Ho, L. et al. An embryonic stem cell chromatin remodeling complex, esBAF, is an essential component of the core pluripotency transcriptional network. Proc. Natl Acad. Sci. USA 106, 5187-5191 (2009).

19. Ho, L. et al. esBAF facilitates pluripotency by conditioning the genome for LIF/STAT3 signalling and by regulating polycomb function. Nat. Cell Biol. 13, 903-913 (2011).

20. Yoo, A. S., Staahl, B. T., Chen, L. \& Crabtree, G. R. MicroRNA-mediated switching of chromatin-remodelling complexes in neural development. Nature 460, 642-646 (2009).

21. Wu, J. I. et al. Regulation of dendritic development by neuron-specific chromatin remodeling complexes. Neuron 56, 94-108 (2007).

22. Lessard, J. et al. An essential switch in subunit composition of a chromatin remodeling complex during neural development. Neuron 55, 201-215 (2007)

23. Versteege, I. et al. Truncating mutations of hSNF5/INI1 in aggressive paediatric cancer. Nature 394, 203-206 (1998)

24. Kadoch, C. \& Crabtree, G. R. Reversible disruption of mSWI/SNF (BAF) complexes by the SS18-SSX oncogenic fusion in synovial sarcoma. Cell 153, 71-85 (2013).

25. Witkowski, L. et al. Germline and somatic SMARCA4 mutations characterize small cell carcinoma of the ovary, hypercalcemic type. Nat. Genet. 46, 438-443 (2014).

26. Le Loarer, F. et al. SMARCA4 inactivation defines a group of undifferentiated thoracic malignancies transcriptionally related to BAF-deficient sarcomas. Nat. Genet. 47, 1200-1205 (2015).

27. Biegel, J. A., Busse, T. M. \& Weissman, B. E. SWI/SNF chromatin remodeling complexes and cancer. Am. J. Med. Genet. C Semin. Med. Genet. 166C, 350-366 (2014).

28. Hoffman, G. R. et al. Functional epigenetics approach identifies BRM/ SMARCA2 as a critical synthetic lethal target in BRG1-deficient cancers. Proc. Natl Acad. Sci. USA 111, 3128-3133 (2014).

29. Puca, L. et al. Patient derived organoids to model rare prostate cancer phenotypes. Nat. Commun. 9, 2404 (2018).

30. Spahn, M. et al. Expression of microRNA-221 is progressively reduced in aggressive prostate cancer and metastasis and predicts clinical recurrence. Int. J. Cancer 127, 394-403 (2010).

31. Prensner, J. R. et al. The long non-coding RNA PCAT-1 promotes prostate cancer cell proliferation through cMyc. Neoplasia 16, 900-908 (2014)

32. Coppola, V. et al. BTG2 loss and miR-21 upregulation contribute to prostate cell transformation by inducing luminal markers expression and epithelialmesenchymal transition. Oncogene 32, 1843-1853 (2013).

33. Ding, Y. et al. Chromatin remodeling ATPase BRG1 and PTEN are synthetic lethal in prostate cancer. J. Clin. Invest. 129, 759-773 (2019).

34. Muthuswami, R. et al. BRG1 is a prognostic indicator and a potential therapeutic target for prostate cancer. J. Cell Physiol. 234, 15194-15205 (2019).

35. Hieronymus, $\mathrm{H}$. et al. Gene expression signature-based chemical genomic prediction identifies a novel class of HSP90 pathway modulators. Cancer Cell 10, 321-330 (2006)

36. Epstein, J. I. et al. A contemporary prostate cancer grading system: a validated alternative to the Gleason score. Eur. Urol. 69, 428-435 (2015).

37. Cancer Genome Atlas Research, N. The molecular taxonomy of primary prostate cancer. Cell 163, 1011-1025 (2015).

38. Spratt, D. E. et al. Individual patient-level meta-analysis of the performance of the decipher genomic classifier in high-risk men after prostatectomy to predict development of metastatic disease. J. Clin. Oncol. 35, 1991-1998 (2017).

39. Ross, A. E. et al. Tissue-based genomics augments post-prostatectomy risk stratification in a natural history cohort of intermediate- and high-risk men. Eur. Urol. 69, 157-165 (2016)

40. Chen, C. D. et al. Molecular determinants of resistance to antiandrogen therapy. Nat. Med. 10, 33-39 (2004).

41. Raupach, E. A. et al. Novel functional insights revealed by distinct proteinprotein interactions of the residual SWI/SNF complex in SMARCA4-deficient small cell carcinoma of the ovary, hypercalcemic type. bioRxiv 794776 (2019). 
42. Chatterjee, S. S., Biswas, M. \& Sengupta, A. Transcriptional cooperativity between SWI/SNF and nurd chromatin remodelers in acute myeloid leukemia. Exp. Hematol. 64, S58 (2018).

43. Yao, J. et al. The homeobox gene, HOXB13, regulates a mitotic protein-kinase interaction network in metastatic prostate cancers. Sci. Rep. 9, 9715 (2019).

44. Artegiani, B. et al. The interaction with $\mathrm{HMG} 20 \mathrm{a} / \mathrm{b}$ proteins suggests a potential role for beta-dystrobrevin in neuronal differentiation. J. Biol. Chem. 285, 24740-24750 (2010).

45. Gupta, K. \& Gupta, S. Neuroendocrine differentiation in prostate cancer: key epigenetic players. Transl. Cancer Res. 6, S104-s108 (2017).

46. Li, Y. et al. SRRM4 drives neuroendocrine transdifferentiation of prostate adenocarcinoma under androgen receptor pathway inhibition. Eur. Urol. 71, 68-78 (2017)

47. Fraser, J. A., Sutton, J. E., Tazayoni, S., Bruce, I. \& Poole, A. V. hASH1 nuclear localization persists in neuroendocrine transdifferentiated prostate cancer cells, even upon reintroduction of androgen. Sci. Rep. 9, 19076 (2019).

48. Zhu, Y. et al. Interleukin-6 induces neuroendocrine differentiation (NED) through suppression of RE-1 silencing transcription factor (REST). Prostate 74, 1086-1094 (2014).

49. Zhang, X. et al. SRRM4 expression and the loss of REST activity may promote the emergence of the neuroendocrine phenotype in castration-resistant prostate cancer. Clin. Cancer Res. 21, 4698-4708 (2015).

50. Chen, R., Li, Y., Buttyan, R. \& Dong, X. Implications of PI3K/AKT inhibition on REST protein stability and neuroendocrine phenotype acquisition in prostate cancer cells. Oncotarget 8, 84863-84876 (2017).

51. Chang, Y. T. et al. REST is a crucial regulator for acquiring EMT-like and stemness phenotypes in hormone-refractory prostate cancer. Sci. Rep. 7, 42795 (2017).

52. Small, E. J. et al. Neuroendocrine prostate cancer in patients with metastatic castration resistant prostate cancer resistant to abiraterone or enzalutamide: preliminary results from the SU2C/PCF/AACR west coast prostate cancer dream team. In ESMO Vol. 25, iv255-iv279 (Annals of Oncology, 2014).

53. Khalaf, D. J. et al. Optimal sequencing of enzalutamide and abiraterone acetate plus prednisone in metastatic castration-resistant prostate cancer: a multicentre, randomised, open-label, phase 2, crossover trial. Lancet Oncol. 20, 1730-1739 (2019).

54. Zou, M. et al. Transdifferentiation as a mechanism of treatment resistance in a mouse model of castration-resistant prostate cancer. Cancer Discov. 7, 736-749 (2017).

55. Zehir, A. et al. Mutational landscape of metastatic cancer revealed from prospective clinical sequencing of 10,000 patients. Nat. Med. 23, 703-713 (2017).

56. Roy, N. et al. Brgl promotes both tumor-suppressive and oncogenic activities at distinct stages of pancreatic cancer formation. Genes Dev. 29, 658-671 (2015).

57. Velcheti, V., Wong, K. K. \& Saunthararajah, Y. EZH2 Inhibitors: take it EZy, it is all about context. Cancer Discov. 9, 472-475 (2019).

58. Sun, X. et al. Aridla has context-dependent oncogenic and tumor suppressor functions in liver cancer. Cancer Cell 32, 574-589 e6 (2017).

59. Jones, S. et al. Frequent mutations of chromatin remodeling gene ARID1A in ovarian clear cell carcinoma. Science 330, 228-231 (2010).

60. Buscarlet, M. et al. Essential role of BRG, the ATPase subunit of BAF chromatin remodeling complexes, in leukemia maintenance. Blood 123, 1720-1728 (2014).

61. $\mathrm{Wu}, \mathrm{Q}$. et al. The BRG1 chromatin remodeling enzyme links cancer cell metabolism and proliferation. Oncotarget 7, 38270-38281 (2016).

62. Hiramatsu, H. et al. The role of the SWI/SNF chromatin remodeling complex in maintaining the stemness of glioma initiating cells. Sci. Rep. 7, 889 (2017).

63. Clark, J. et al. Identification of novel genes, SYT and SSX, involved in the $t$ $(\mathrm{X} ; 18)(\mathrm{p} 11.2 ; \mathrm{q} 11.2)$ translocation found in human synovial sarcoma. Nat. Genet. 7, 502-508 (1994).

64. Jubierre, L. et al. BRG1/SMARCA4 is essential for neuroblastoma cell viability through modulation of cell death and survival pathways. Oncogene 35, 5179-5190 (2016).

65. Laurette, P. et al. Chromatin remodellers Brg1 and Bptf are required for normal gene expression and progression of oncogenic Braf-driven mouse melanoma. Cell Death Differ. 27, 29-43 (2019).

66. Baca, S. C. \& Garraway, L. A. The genomic landscape of prostate cancer. Front. Endocrinol. 3, 69 (2012)

67. Barbieri, C. E. et al. Exome sequencing identifies recurrent SPOP, FOXA1 and MED12 mutations in prostate cancer. Nat. Genet. 44, 685-689 (2012).

68. Armenia, J. et al. The long tail of oncogenic drivers in prostate cancer. Nat. Genet. 50, 645-651 (2018).

69. Link, K. A. et al. Targeting the BAF57 SWI/SNF subunit in prostate cancer: a novel platform to control androgen receptor activity. Cancer Res. 68 4551-4558 (2008).

70. Jin, M. L., Kim, Y. W. \& Jeong, K. W. BAF53A regulates androgen receptormediated gene expression and proliferation in LNCaP cells. Biochem. Biophys. Res. Commun. 505, 618-623 (2018).
71. Sandoval, G. J. et al. Binding of TMPRSS2-ERG to BAF chromatin remodeling complexes mediates prostate oncogenesis. Mol. Cell 71, 554-566 e7 (2018).

72. Prensner, J. R. et al. The long noncoding RNA SChLAP1 promotes aggressive prostate cancer and antagonizes the SWI/SNF complex. Nat. Genet. 45, 1392-1398 (2013)

73. Raab, J. R. et al. SWI/SNF remains localized to chromatin in the presence of SCHLAP1. Nat. Genet. 51, 26-29 (2019).

74. Sun, A. et al. Aberrant expression of SWI/SNF catalytic subunits BRG1/BRM is associated with tumor development and increased invasiveness in prostate cancers. Prostate 67, 203-213 (2007).

75. Shen, H. et al. The SWI/SNF ATPase Brm is a gatekeeper of proliferative control in prostate cancer. Cancer Res. 68, 10154-10162 (2008).

76. Labrecque, M. P. et al. Molecular profiling stratifies diverse phenotypes of treatment-refractory metastatic castration-resistant prostate cancer. J. Clin. Invest. 130, 4492-4505 (2019).

77. Lazzaro, D., Price, M., de Felice, M. \& Di Lauro, R. The transcription factor TTF-1 is expressed at the onset of thyroid and lung morphogenesis and in restricted regions of the foetal brain. Development 113, 1093-1104 (1991).

78. Sussel, L., Marin, O., Kimura, S. \& Rubenstein, J. L. Loss of Nkx2.1 homeobox gene function results in a ventral to dorsal molecular respecification within the basal telencephalon: evidence for a transformation of the pallidum into the striatum. Development 126, 3359-3370 (1999).

79. Minocha, S. \& Herr, W. Cortical and commissural defects upon HCF-1 loss in Nkx2.1-derived embryonic neurons and glia. Dev. Neurobiol. 79, 578-595 (2019).

80. Hofer, M. D. et al. The role of metastasis-associated protein 1 in prostate cancer progression. Cancer Res. 64, 825-829 (2004).

81. Norris, J. D. et al. The homeodomain protein HOXB13 regulates the cellular response to androgens. Mol. Cell 36, 405-416 (2009).

82. Chen, $Z$. et al. Diverse AR-V7 cistromes in castration-resistant prostate cancer are governed by HoxB13. Proc. Natl Acad. Sci. USA 115, 6810-6815 (2018).

83. Ewing, C. M. et al. Germline mutations in HOXB13 and prostate-cancer risk N. Engl. J. Med. 366, 141-149 (2012).

84. Abeshouse, A. et al. The molecular taxonomy of primary prostate. Cancer Cell 163, 1011-1025 (2015).

85. Talevich, E., Shain, A. H., Botton, T. \& Bastian, B. C. CNVkit: genome-wide copy number detection and visualization from targeted DNA sequencing. PLoS Comput. Biol. 12, e1004873 (2016).

86. Cibulskis, K. et al. Sensitive detection of somatic point mutations in impure and heterogeneous cancer samples. Nat. Biotechnol. 31, 213 (2013).

87. McLaren, W. et al. The ensembl variant effect predictor. Genome Biol. 17, 122 (2016).

88. Prandi, D. et al. Unraveling the clonal hierarchy of somatic genomic aberrations. Genome Biol. 15, 439 (2014).

89. Beltran, H. et al. Molecular characterization of neuroendocrine prostate cancer and identification of new drug targets. Cancer Disco. 1, 487-495 (2011).

90. Chakravarty, D. et al. The oestrogen receptor alpha-regulated lncRNA NEAT1 is a critical modulator of prostate cancer. Nat. Commun. 5, 5383 (2014).

91. Dobin, A. et al. STAR: ultrafast universal RNA-seq aligner. Bioinformatics 29, 15-21 (2012)

92. Habegger, L. et al. RSEQtools: a modular framework to analyze RNA-Seq data using compact, anonymized data summaries. Bioinformatics 27, 281-283 (2010).

93. Colaprico, A. et al. TCGAbiolinks: an R/Bioconductor package for integrative analysis of TCGA data. Nucleic Acids Res. 44, e71 (2016).

94. Piccolo, S. R. et al. A single-sample microarray normalization method to facilitate personalized-medicine workflows. Genomics 100, 337-344 (2012)

95. Dobin, A. et al. STAR: ultrafast universal RNA-seq aligner. Bioinformatics 29, 15-21 (2013)

96. Nikolayeva, O. \& Robinson, M. D. edgeR for differential RNA-seq and ChIP seq analysis: an application to stem cell biology. Methods Mol. Biol. 1150 45-79 (2014).

97. Subramanian, A. et al. Gene set enrichment analysis: a knowledge-based approach for interpreting genome-wide expression profiles. Proc. Natl Acad. Sci. USA 102, 15545-15550 (2005).

98. Liberzon, A. et al. Molecular signatures database (MSigDB) 3.0. Bioinformatics 27, 1739-1740 (2011).

99. Rappsilber, J., Ishihama, Y. \& Mann, M. Stop and go extraction tips for matrix-assisted laser desorption/ionization, nanoelectrospray, and LC/MS sample pretreatment in proteomics. Anal. Chem. 75, 663-670 (2003).

100. Elias, J. E. \& Gygi, S. P. Target-decoy search strategy for increased confidence in large-scale protein identifications by mass spectrometry. Nat. Methods 4, 207-214 (2007).

101. Huttlin, E. L. et al. A tissue-specific atlas of mouse protein phosphorylation and expression. Cell 143, 1174-1189 (2010).

102. Tyanova, S. et al. The Perseus computational platform for comprehensive analysis of (prote)omics data. Nat. Methods 13, 731-740 (2016). 


\section{Acknowledgements}

We thank patients and their families for participating in genomics, transcriptomics, and precision cancer care studies. The authors would like to thank Cigall Kadoch from Dana Farber Cancer Institute for her important insights into SWI/SNF biology. We thank current and previous members of the Rubin lab for valuable discussions, Charles Sawyers and Ping Mu at Memorial Sloan-Kettering Cancer Center for sharing the LNCaP-AR cell line. We are grateful to Juan Miguel Mosquera, Brian Robinson, and Verena Sailer for their prostate cancer tissue contributions. We are thankful for expert assistance from the Translational Research Program at WCMC Pathology and Laboratory Medicine (Bing He, Leticia Dizon, Yifang Liu, Mai Ho) and the WCM CLC Genomics Core Facility (Jenny Xiang). We thank Inti Zlobec and Micha D. Eichmann at the University of Bern for their assistance with the Scorenado TMA scoring platform. We thank Elai Davicioni (Decipher Biosciences, Cam, USA) for providing data for gene expression analyses. We further thank the Next Generation Platform (Institute of Genetics, Bern) and Irene Keller (DBMR, Bern) for their assistance with the single cell experiments. We acknowledge expert assistance from Mariana Ricca at the University of Bern in preparing the manuscript for submission. This project has received funding from the Nuovo-Soldati Foundation (J.C.), the NIH/NCI WCM SPORE in Prostate Cancer P50-CA211024 (V.C., H.B., K.B., F.D., M.A.R.), the Swiss National Science Foundation Project grants number 310030_189149 and 31003A_169352 (M.K.-d.J.) and Ambizione grant number: PZ00P3_168165 (S.P.), the NCI grants number 1R01CA233650 (P.C.), 1R01CA233650 (N.D.), R37 CA241486 (H.B.), and R01 CA125612 (F.D.) and the European Research Council (ERC) under the European Union's Horizon 2020 research and innovation programme (grant agreement No 648670) (F.D.).

\section{Author contributions}

J.C., A.A., and M.A.R. designed the study and the experiments. J.C., A.A., P.T., D.W., S.-S.C., S.C., M.J., and L.B. performed experiments. M.R.d.F., S.P., D.P., M.B., and F.D. performed genomic and transcriptomic analyses of patient data. M.R.d.F., S.P., R.B., and A.S. performed RNA-seq analysis of experimental data. L.R. and S.P. performed scRNAseq and ChIP-seq analysis. M.R.d.F., S.P., and M.A. performed analyses on Decipher GRID and JHMI cohorts. V.C. and K.V.B. performed survival analyses. J.C. and M.A.R. performed pathology review and immunohistochemical evaluation. P.C. and N.D. performed mass spectrometry experiments and analyzed respective data. A.-C.U., S.B.L. performed mass spectrometry analyses. F.F., T.L.L., M.S. and M.K.-d.J. established annotated patient cohorts and provided clinical data. H.B. provided CRPC-NE patient samples, L.P., S.W., and Y.C. established patient-derived organoid models. M.A.R. provided administrative, technical and material support. J.C., A.A., and M.A.R. wrote the initial draft of the manuscript and all authors contributed to the final version.

\section{Competing interests}

H.B. has received research funding from Janssen, Astellas, Abbvie, Millennium, and Eli Lilly and consulting with Janssen, Astellas, Sanofi Genzyme, Astra Zeneca, Pfizer. L.P. is now an employee of Loxo Oncology at Lilly. M.A.R. has received research funding from Novartis, Roche, Ventana, Janssen, Astellas, Millennium, and Eli Lilly. M.A.R. is on the $\mathrm{SAB}$ of Neogenomics. T.L.L. has received research funding from DeepBio, Decipher, Ventana/Roche. Cornell and Bern Universities have filed a patent application on SWI/ SNF diagnostic and therapeutic fields with A.A., J.C., and M.A.R. listed as inventors. The remaining authors declare no competing interests.

\section{Additional information}

Supplementary information is available for this paper at https://doi.org/10.1038/s41467020-19328-1.

Correspondence and requests for materials should be addressed to M.A.R.

Peer review information Nature Communications thanks Gong-Hong Wei and the other, anonymous, reviewer(s) for their contribution to the peer review of this work. Peer reviewer reports are available.

Reprints and permission information is available at http://www.nature.com/reprints

Publisher's note Springer Nature remains neutral with regard to jurisdictional claims in published maps and institutional affiliations.

Open Access This article is licensed under a Creative Commons Attribution 4.0 International License, which permits use, sharing, adaptation, distribution and reproduction in any medium or format, as long as you give appropriate credit to the original author(s) and the source, provide a link to the Creative Commons license, and indicate if changes were made. The images or other third party material in this article are included in the article's Creative Commons license, unless indicated otherwise in a credit line to the material. If material is not included in the article's Creative Commons license and your intended use is not permitted by statutory regulation or exceeds the permitted use, you will need to obtain permission directly from the copyright holder. To view a copy of this license, visit http://creativecommons.org/ licenses/by/4.0/

(C) The Author(s) 2020

Joanna Cyrta1,2,29, Anke Augspach ${ }^{1,29}$, Maria Rosaria De Filippo ${ }^{3,4}$, Davide Prandi ${ }^{5}$, Phillip Thienger ${ }^{1}$, Matteo Benelli ${ }^{5,6}$, Victoria Cooley ${ }^{7}$, Rohan Bareja ${ }^{2,8}$, David Wilkes (1) ${ }^{2}$, Sung-Suk Chae ${ }^{9}$, Paola Cavaliere ${ }^{10}$, Noah Dephoure ${ }^{10,11}$, Anne-Christine Uldry ${ }^{12}$, Sophie Braga Lagache ${ }^{12}$, Luca Roma ${ }^{4}$, Sandra Cohen ${ }^{9}$, Muriel Jaquet', Laura P. Brandt ${ }^{1}$, Mohammed Alshalalfa (1) ${ }^{13}$, Loredana Puca ${ }^{14}$, Andrea Sboner 2,8,15,16, Felix Feng (1D ${ }^{12}$, Shangqian Wang ${ }^{17}$, Himisha Beltran (1) ${ }^{14,18}$, Tamara Lotan 19,20,21, Martin Spahn22,23, Marianna Kruithof-de Julio (iD) 1,3,24, Yu Chen (10 ${ }^{14}$, Karla V. Ballman7, Francesca Demichelis (iD ${ }^{2,5}$, Salvatore Piscuoglio ${ }^{4,25,26,30}$ \& Mark A. Rubin (1) ${ }^{1,27,28,30 \times}$

${ }^{1}$ Department for BioMedical Research, University of Bern, 3008 Bern, Switzerland. ${ }^{2}$ The Caryl and Israel Englander Institute for Precision Medicine, Weill Cornell Medicine, New York, NY 10021, USA. ${ }^{3}$ Department for BioMedical Research, Urology Research Laboratory, University of Bern, 3008 Bern, Switzerland. ${ }^{4}$ Institute of Pathology and Medical Genetics, University Hospital Basel, University of Basel, 4051 Basel, Switzerland.

${ }^{5}$ Department of Cellular, Computational and Integrative Biology (CIBIO), University of Trento, 38122 Trento, Italy. ${ }^{6}$ Bioinformatics Unit, Hospital of Prato, 59100 Prato, Italy. ${ }^{7}$ Department of Healthcare Policy and Research, Division of Biostatistics and Epidemiology, Weill Cornell Medicine, New York, NY 10021, USA. ${ }^{8}$ Institute for Computational Biomedicine, Weill Cornell Medicine, New York, NY 10021, USA. ${ }^{9}$ Department of Laboratory Medicine and Pathology, Weill Cornell Medicine, New York, NY 10021, USA. ${ }^{10}$ Meyer Cancer Center, Weill Cornell Medicine, New York, NY 10021, USA. ${ }^{11}$ Department of Biochemistry, Weill Cornell Medicine, New York, NY 10021, USA. ${ }^{12}$ Proteomics Mass Spectrometry Core Facility, University of Bern, 3010 Bern, Switzerland. ${ }^{13}$ Department of Radiation Oncology, Helen Diller Family Comprehensive Cancer Center, University of California at San Francisco, San Francisco, CA, USA. ${ }^{14}$ Department of Medicine, Division of Medical Oncology, Weill Cornell Medicine, New York, NY 10021, USA. ${ }^{15} \mathrm{HRH}$ Prince Alwaleed Bin Talal Bin Abdulaziz Alsaud Institute for Computational Biomedicine, Weill Cornell Medicine, New York, NY 10021, USA. ${ }^{16}$ Meyer Cancer Center, Weill Cornell Medicine, New York, NY 10065, USA. ${ }^{17}$ Human Oncology and Pathogenesis Program and Department of Medicine, Memorial Sloan-Kettering Cancer Center, New York, NY 10065, USA. ${ }^{18}$ Department of Medical Oncology, Dana Farber Cancer 
Institute, Boston, MA, USA. ${ }^{19}$ Department of Urology, Johns Hopkins University School of Medicine, Baltimore, Maryland, USA. ${ }^{20}$ Department of Pathology, Johns Hopkins University School of Medicine, Baltimore, MD 21205, USA. ${ }^{21}$ Department of Oncology, Johns Hopkins University School of Medicine, Baltimore, MD 21205, USA. ${ }^{22}$ Lindenhofspital Bern, Prostate Center Bern, 3012 Bern, Switzerland. ${ }^{23}$ Department of Urology, Essen University Hospital, University of Duisburg-Essen, 47057 Essen, Germany. ${ }^{24}$ Department of Urology, Inselspital, 3010 Bern, Switzerland. ${ }^{25}$ Visceral Surgery Research Laboratory, Clarunis, Department of Biomedicine, University of Basel, 4051 Basel, Switzerland. ${ }^{26}$ Clarunis Universitäres Bauchzentrum Basel, 4002 Basel, Switzerland. ${ }^{27}$ Inselspital, 3010 Bern, Switzerland. ${ }^{28}$ Bern Center for Precision Medicine, 3008 Bern, Switzerland. ${ }^{29}$ These authors contributed equally: Joanna Cyrta, Anke Augspach. ${ }^{30}$ These authors jointly supervised this work: Salvatore Piscuoglio, Mark A. Rubin. ${ }_{\text {email: mark.rubin@dbmr.unibe.ch }}$ 